\title{
Cosmic rays and the magnetic field in the nearby starburst galaxy NGC 253 III. Helical magnetic fields in the nuclear outflow
}

\author{
V. Heesen ${ }^{1}$, R. Beck ${ }^{2}$, M. Krause ${ }^{2}$, and R.-J. Dettmar ${ }^{3}$ \\ ${ }^{1}$ Centre for Astrophysics Research, University of Hertfordshire, Hatfield AL10 9AB, UK \\ e-mail: v.heesen@herts .ac.uk \\ 2 Max-Planck-Institut für Radioastronomie, Auf dem Hügel 69, 53121 Bonn, Germany \\ 3 Astronomisches Institut der Ruhr-Universität Bochum, Universitätsstr. 150, 44780 Bochum, Germany
}

Received 1 July 2011 / Accepted 30 August 2011

\begin{abstract}
Context. Magnetic fields are good tracers of gas compression by shock waves in the interstellar medium. These can be caused by the interaction of star-formation driven outflows from individual star formation sites as described in the chimney model. Integration along the line-of-sight and cosmic-ray diffusion may hamper detection of compressed magnetic fields in many cases.

Aims. We study the magnetic field structure in the central part of the nuclear starburst galaxy NGC 253 with spatial resolutions between 40 and $150 \mathrm{pc}$ to detect any filamentary emission associated with the nuclear outflow. As the nuclear region is much brighter than the rest of the disc we can distinguish this emission from that of the disc.

Methods. We used radio polarimetric observations with the VLA. New observations at $\lambda 3 \mathrm{~cm}$ with $77^{\prime \prime} 5$ resolution were combined with archive data at $\lambda \lambda 20$ and $6 \mathrm{~cm}$. We created a map of the rotation measure distribution between $\lambda \lambda 6$ and $3 \mathrm{~cm}$ and compared it with a synthetic polarization map.

Results. We find filamentary radio continuum emission in a geometrical distribution, which we interpret as the boundary of the NW nuclear outflow cone seen in projection. The scaleheight of the continuum emission is $150 \pm 20 \mathrm{pc}$, regardless of the observing frequency. The equipartition magnetic field strength is $46 \pm 10 \mu \mathrm{G}$ for the total field and $21 \pm 5 \mu \mathrm{G}$ for the regular field in the filaments. We find that the ordered magnetic field is aligned along the filaments, in agreement with amplification due to compression. The perpendicular diffusion coefficient across the filaments is $\kappa_{\perp}=1.5 \times 10^{28} \mathrm{~cm}^{2} \mathrm{~s}^{-1} \cdot E(\mathrm{GeV})^{0.5 \pm 0.7}$. In the SE part of the nuclear outflow cone the magnetic field is pointing away from the disc in form of a helix, with an azimuthal component increasing up to at least $1200 \mathrm{pc}$ height, where it is about equal to the total component. The ordered magnetic field in the disc is anisotropic within a radius of $2.2 \mathrm{kpc}$. At larger radii, the large-scale field is regular and of even parity.

Conclusions. The magnetic filaments indicate an interaction of the nuclear outflow with the interstellar medium. The magnetic field is able to collimate the outflow, which can explain the observed small opening angle of $\approx 26^{\circ}$. Owing to the conservation of angular momentum by the plasma in the nuclear outflow, the field lines are frozen into the plasma, and they wind up into a helix. Strong adiabatic losses of the cosmic-ray electrons in the accelerated outflow can partly explain why the radio luminosity of the nucleus lies below the radio-FIR correlation.
\end{abstract}

Key words. magnetic fields - galaxies: magnetic fields - galaxies: ISM - cosmic rays - galaxies: individual: NGC 253 methods: observational

\section{Introduction}

Magnetic fields are an important ingredient of the interstellar medium. Not only do they facilitate star formation, but they also make an important contribution to the energy budget. The global magnetic field structure of galaxies can be described by a spiral field in the disc and a poloidal field in the halo, which takes an $\mathrm{X}$-shape when seen edge-on (Krause 2008; Beck 2009; Braun et al. 2010). Such X-shaped structures may be formed by the hydrodynamic $\mathrm{Al}$ outflows driven by star formation in the disc (Dalla Vecchia \& Schaye 2008). On the other hand, a concurring theory is that these magnetic field structures are part of a largescale field, possibly generated by a galactic dynamo (Gissinger et al. 2009; Hanasz et al. 2009). Therefore, it is desirable to understand how the dynamics of the interstellar medium influence the structure of the magnetic field that is frozen into the gas. The magnetic field may only be a tracer for interactions but can also influence the dynamics if its energy density is high enough.
Observations of nearby galaxies show that the large-scale magnetic field is a very sensitive tracer for interaction in the interstellar medium that is not seen at any other wavelength. An example for this are the colliding antenna galaxies that show a maximum of polarized intensity outside of their optical extents (Chyży \& Beck 2004). Several galaxies in the Virgo cluster show signs of interaction possibly due to ram pressure compression as sharp edges in the total power emission and enhanced polarization (Vollmer et al. 2007, 2010; Weżgowiec et al. 2007). MHD simulations by de Avillez (2000) of the interstellar medium reveal a highly filamentary disc-halo interface with transient vertical filaments in the gas and magnetic fields. The filaments could be regarded as the walls of so-called chimneys through which hot gas can break out from the disc into the halo when a sufficient number of supernovae occur in a OB association (Norman \& Ikeuchi 1989; Mac Low \& Ferrara 1999). Farther up in the halo the gas flow may be driven 
by the cosmic-ray pressure, which has a larger scaleheight and is not so much affected by radiation losses (Ipavich 1975; Breitschwerdt et al. 1991; Breitschwerdt 2008). Observations of this phenomenon will be left to the upcoming radio telescopes that can observe at the very lowest frequencies and are sensitive to the aged cosmic-ray electrons. Magnetic fields may, however, already be important for outflows in the disc.

Searches for small-scale magnetic field structures in galaxies that trace the filamentary disc-halo interface are rare. Normally the radio continuum emission is smooth and can be described as two-component exponential haloes with a thin $(300 \mathrm{pc})$ and a thick radio (1.8 kpc) disc (Dumke \& Krause 1998). Vertical filaments are observed in M 82 with prominent gaps in the continuum emission, possibly created by vertical magnetic fields, which prevent horizontal diffusion (Reuter et al. 1992, 1994). But it was not possible to relate these minima to a particular outflow cone (Wills et al. 1999). In NGC 5775 vertical filaments in the radio spectral index suggest the outflow of young cosmicray electrons, but they do not coincide with the observed radio spurs with vertical magnetic fields (Duric et al. 1998; Tüllmann et al. 2000). In NGC 1569 a huge $\mathrm{H} \alpha$ bubble can be identified as the edge of a radio spur (Kepley et al. 2010). In NGC 253 the halo magnetic field seems to line up tangentially with the edge of the superbubble filled with soft X-ray emission (Heesen et al. 2009b, hereafter Paper II).

NGC 253 is an archetypical starburst galaxy that has both a superwind and a disc wind (Heckman et al. 2000; Strickland et al. 2002; Heesen et al. 2009a). The superwind is traced by the hot X-ray emission seen in the halo, indicating the outflow of hot gas, possibly supernova-heated. The hot gas is seen in the centre as a plume of $600 \mathrm{pc}$ length emerging to the southeastern (SE) side (Strickland et al. 2000; Bauer et al. 2007). The northwestern (NW) side is on the far side of the disc and thus heavily absorbed in X-ray emission (Pietsch et al. 2000). Bauer et al. (2007) detected with sensitive XMM-Newton observations the X-ray emission of the actual fluid in the outflow, whereas Strickland et al. (2000) used the superior resolution of the Chandra telescope to resolve the filaments consisting of cooled material. The speed of the hot gas could be measured from $\mathrm{H} \alpha$ emission lines to be $390 \mathrm{~km} \mathrm{~s}^{-1}$ by Schulz \& Wegner (1992). Recent, optical integral-field unit, spectroscopy observations could measure the increase in the outflow velocity with increasing distance from the nucleus (Westmoquette et al. 2011).

The disc wind that is traced by a radio continuum halo is different from the nuclear outflow. In Heesen et al. (2009a, hereafter Paper I), we have shown that the cosmic-ray electrons are transported with a bulk velocity of about $300 \mathrm{~km} \mathrm{~s}^{-1}$ in a mainly vertical direction over the full extent of the disc. The outflow speed varies only slightly as a function of the galactocentric radius, further supporting the idea of a disc wind, because it seems unrelated to the nuclear outflow. A high-resolution study of the continuum emission by Zirakashvili \& Völk (2006) of the inner disc showed a very similar outflow speed.

In Paper II we investigated the magnetic field structure and found that it is an axisymmetric spiral in the disc and X-shaped in the halo, as in several other edge-on galaxies (Krause 2008). The origin of the X-shapes is still not clear, but in NGC 253 it may be related to the interaction of the disc wind with the superwind. Simulations of a superwind by Suchkov et al. (1994) show the formation of huge bubbles. The outer lobes of the bubbles show an X-shaped structure in projection. If the large-scale magnetic field is compressed in these lobes and aligned with them, they would also form an X-shaped structure.
So far, no high-resolution magnetic field maps have been available for NGC 253 that would allow us to trace the structure of the X-shaped halo field back into the inner disc and study its interaction with the nuclear outflow. The magnetic field maps shown in Paper II have a resolution of 30". In this paper, we present new observations with less than $10^{\prime \prime}$ resolution to study the magnetic field structure in the inner disc of NGC 253. In Sect. 2 we present our observations and data reduction. In Sect. 3 we discuss the structure of the radio continuum emission. Section 4 contains the analysis of the magnetic field structure measured with polarimetry. Section 5 contains the discussion and Sect. 6 our conclusions. In this paper we use the distance from Karachentsev et al. (2003) of 3.94 Mpc $\left(1^{\prime \prime}=19.1 \mathrm{pc}\right)$, a position angle of $52^{\circ}$, and an inclination angle of $78^{\circ}$ (Pence 1981).

\section{Observations and data reduction}

\subsection{New VLA observations}

We used radio continuum polarimetric observations with the Very Large Array (VLA) at $\lambda 3 \mathrm{~cm}$ with a bandwidth of $2 \times 50 \mathrm{MHz}^{1}$. The observations were carried out in October 2009 on three days amounting to $11 \mathrm{~h}$ of total observing time. We used a hybrid DnC-configuration to get a circular beam at the southern declination of NGC $253\left(\delta=-25^{\circ}\right)$. We selected three pointings with about $3.5 \mathrm{~h}$ of on-source integration time each. The data were calibrated with $\mathrm{J} 0137+331$ (3C 48) using the Baars et al. (1977) flux scale, and J0025-260 was used as a secondary (phase) calibrator.

For the data reduction we used the Astronomical Image Processing System (AIPS) ${ }^{2}$. For total power (Stokes $I$ ), the dynamic range has to be large because of the 1 Jy nuclear point-like source. We used self-calibration first with only phase solutions $(2 \times)$ and subsequently with a solution in phase \& amplitude $(2 \times)$, which improved our dynamic range. Of the 24 antennas in the array, 21 were upgraded EVLA antennas and three were VLA antennas. Our final map improved significantly when we left out the three VLA antennas, which we did not investigate any further. The rms noise level of $50 \mu \mathrm{Jy}_{\text {beam }^{-1}}$ is a factor of 3 higher than the theoretically expected value. We explain this with the high dynamic range, where in our map the peak-to-noise ratio is 13000 . Since the sensitivity is enough for our analysis and the polarization is not affected by it, as shown below, we did not try to improve our map any further.

The polarization leakage (D-terms) was calibrated with PCAL using the secondary calibrator and observing the variation in the polarization as a function of the parallactic angle. The source intrinsic polarization varies with the parallactic angle, whereas the instrumental polarization stays constant, allowing us to separate them from each other. The polarization angle is calibrated with the known angle of $3 \mathrm{C} 48$. The calibrated $(u, v)$-data were inverted and cleaned until we reached a level of $2 \times$ the rms noise level. We used a robust weighting (Briggs 1995) with robust $=0$, which gave us a beam size of 7 '.5 both in total power and in polarization $^{3}$. The three pointings were combined with LTESS (part of AIPS) where a linear superposition is used with a correction for the attenuation of the primary beam (Braun 1988).

\footnotetext{
1 The National Radio Astronomy Observatory (NRAO) is a facility of the National Science Foundation operated under cooperative agreement by Associated Universities, Inc.

2 AIPS is free software from NRAO.

3 All resolutions are referred to as the half power beam width (HPBW).
} 
Table 1. VLA observations presented in this paper.

\begin{tabular}{|c|c|c|c|c|c|c|c|c|c|}
\hline $\begin{array}{l}\lambda \\
{[\mathrm{cm}]}\end{array}$ & $\begin{array}{c}v \\
{[\mathrm{GHz}]}\end{array}$ & Array & Resolution & $\begin{array}{c}\text { TP noise } \\
{[\mu \mathrm{Jy} \mathrm{b}}\end{array}$ & $\begin{array}{l}\text { PI noise } \\
\left.\mathrm{am}^{-1}\right]\end{array}$ & $\begin{array}{l}\text { Dynamic } \\
\text { range }\end{array}$ & Date & Code & References \\
\hline 20 & 1.49 & A & $1 . .3 \times 2 . .2$ & 50 & - & 3600 & Jul. 1987 & AU30 & Ulvestad \& Antonucci (1997) \\
\hline 20 & 1.56 & $\mathrm{~B}+\mathrm{C}$ & 7.5 & 400 & - & 2500 & Sep. 1990 & AC278 & Carilli et al. (1992) \\
\hline $6^{*}$ & 4.71 & $\mathrm{C}$ & 7.'5 & 100 & 40 & 8300 & Jan. 1991 & AC278 & this paper \\
\hline $3^{*}$ & 8.46 & $\mathrm{DnC}$ & 7.'5 & 50 & 16 & 13000 & Oct. 2009 & AH899 & this paper \\
\hline
\end{tabular}

Notes. ${ }^{(*)}$ The observations at $\lambda \lambda 6$ and $3 \mathrm{~cm}$ were merged with Effelsberg single-dish data.

We took the part of the maps into account that was above $7 \%$ of the normalized amplitude with respect to the centre of the primary beam. For the two pointings adjacent to the nucleus, we subtracted the nucleus in each "snapshot" of short integration time to remove instrumental effects as the nucleus moves through the primary beam. This procedure gave us an rms noise level of $16 \mu \mathrm{Jybeam}^{-1}$ in Stokes $Q$ and $U$, in agreement with the theoretically expected value, where the maps are largely free of spurious emission from the nucleus.

The $(u, v)$-plane is not filled below a baseline of $30 \mathrm{~m}$ corresponding to a $(u, v)$-distance of $1 \mathrm{k} \lambda$ at $\lambda 3 \mathrm{~cm}$. Therefore, any structures with an angular diameter larger than $3^{\prime}$ cannot be imaged. To reconstruct the large angular scales, we merged the VLA and Effelsberg data using IMERG, which is part of AIPS. The Effelsberg maps were presented in the two preceding papers of this series. The merging was done by a Fourier transform of the two maps to be combined, where an overlap in the $(u, v)$-plane had to be specified as uvrange, so that the observations could be matched. We compared the integrated flux densities in several separated regions in the Stokes $I$ map both, in the disc and halo. The flux densities agreed within $10 \%$ in each of the regions for the chosen overlap of $0-1.2 \mathrm{k} \lambda$. The $Q$ and $U$ maps were merged using the same optimum uvrange as found for $I$.

\subsection{VLA archive data}

Archive observations at $\lambda 6 \mathrm{~cm}$ in the C-configuration are available that have not been published so far. They consist of five pointings along the major axis with $30 \mathrm{~min}$ total integration time each, but spread over $6 \mathrm{~h}$ to have a good coverage in the $(u, v)$ plane. The data were calibrated as described for the $\lambda 3 \mathrm{~cm}$ observations in Stokes $I$ and polarization. As we only have one pointing centred on the nucleus there are no sidelobes of spurious polarization. We inverted the $(u, v)$-data using robust weighting (robust $=0$ ) and cleaned down to $2 \times$ the rms noise level. The map was merged with Effelsberg single-dish data using IMERG, with an overlap of $0.2-0.8 \mathrm{k} \lambda$. The rms noise level in polarization is $40 \mu \mathrm{Jy}_{\text {beam }^{-1}}$, in agreement with what we would expect. In total power the rms noise level is $100 \mu \mathrm{Jy}_{\text {beam }}^{-1}$, limited by the high dynamic range of 8300 due to the bright nuclear source.

Observations in the B-configuration at $\lambda 20 \mathrm{~cm}$ were also taken from archive data. These were already presented in Carilli et al. (1992) albeit with lower resolution, because they were combined with a more compact configuration. We attempted a polarization calibration, but this was not possible, indicated by a large variation in the polarization angle of the primary calibrator with baseline and time. For a successful polarization calibration, the variation should only be a few degrees at most. The observations were centred on the nucleus, where one pointing is enough to cover the central part of the galaxy. We combined the $\lambda 20 \mathrm{~cm}$ observations in B-configuration in total intensity with those in
C-configuration, so that we are sensitive to angular scales of up to $15^{\prime}$, i.e. to all but the largest angular scales. The rms noise level is, with $400 \mu \mathrm{Jy}_{\text {beam }}^{-1}$, slightly higher than in the map presented by Carilli et al. (1992), which has a noise level of $350 \mu \mathrm{Jy} \mathrm{beam}^{-1}$ at $30^{\prime \prime}$ resolution (Paper I).

Furthermore, there is some high-resolution data at $\lambda 20 \mathrm{~cm}$ in VLA A-configuration that was presented in Ulvestad \& Antonucci (1997), although they did not study the extended emission. The data were calibrated in the standard fashion in AIPS where we used a model for the primary calibrator 3C 48, because it is resolved on the longest baselines. We cleaned the $(u, v)$-data using robust weighting (robust $=0)$ to $2 \times$ the $\mathrm{rms}$ noise level. The final noise level is $50 \mu \mathrm{Jy} /$ beam at a resolution of 2 '. $2 \times 1$ '. $3\left(\mathrm{PA}=-2^{\circ}\right)$, which agrees with Ulvestad \& Antonucci (1997). We attempted a polarization calibration of the data, but it failed again, as indicated by the large variation in the primary calibrator's polarization angle. The details of the maps presented in this paper are summarized in Table 1.

For the analysis of the rotation measure we also created natural weighted maps at $\lambda \lambda 6 \mathrm{~cm}$ and $3 \mathrm{~cm}$ with $10^{\prime} .5$ resolution. At $\lambda 6 \mathrm{~cm}$ we combined the data in polarization from our previously published observations in D-configuration with those in C-configuration. At both wavelengths the maps were merged with Effelsberg single-dish data.

\section{Morphology of the radio continuum emission}

\subsection{Nuclear region and bar}

In Fig. 1 we show the $\lambda 20 \mathrm{~cm}$ total power radio continuum distribution overlaid on an $\mathrm{H} \alpha$ image by Hoopes et al. (1996). The radio continuum (though mostly non-thermal) traces the $\mathrm{H}$ II regions well, which are visible as clumps in the $\mathrm{H} \alpha$ image. They form a ring around the centre, where the spiral arm NW of the nucleus is prominent. We discover a long structure in the inner region, extending both NE and SW of the nucleus, resembling a radio continuum bar that has so far not been reported for this galaxy. This radio bar has a position angle of $63^{\circ} \pm 1^{\circ}$. In Fig. 2 we overlayed the $\lambda 6 \mathrm{~cm}$ continuum emission onto a 2MASS $K$-band image that shows the stellar bar (Jarrett et al. 2003). The radio and the stellar bar have about the same length, and they align well, but the former is shifted clockwise with respect to the latter (the stellar bar has a position angle of $70^{\circ} \pm 1^{\circ}$ ).

The galaxy rotates clockwise with trailing spiral arms. According to HI observations in the barred galaxies NGC 1097 and NGC 1365 bar patterns rotate more slowly than the gas (Ondrechen et al. 1989; Ondrechen \& van der Hulst 1989), so that the gas flow enters the bar from behind; i.e. the bar is overtaken by the gas. Therefore the radio bar is downstream of the stellar bar and on the leading edge with respect to the rotation. This is the same behaviour as in the prominent barred galaxy NGC 1365 that has been extensively studied in radio continuum 


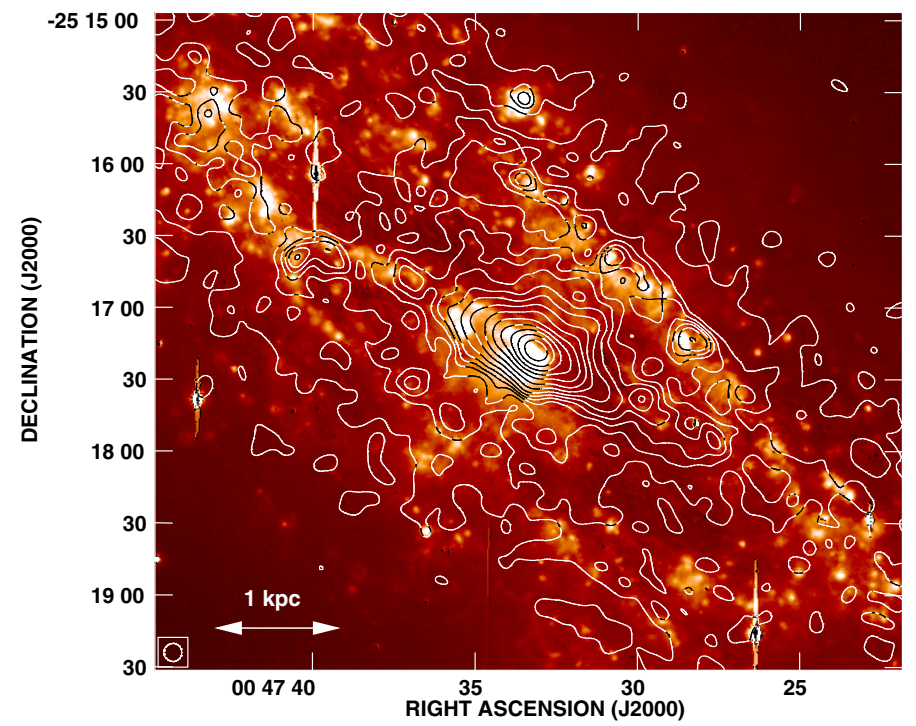

Fig. 1. Total power radio continuum at $\lambda 20 \mathrm{~cm}$ with $7 . .5$ resolution. Contours are at 3, 6, 8, 10, 12, 15, 20, 30, 50, 100, 200, 400, 800, and $1600 \times 400 \mu \mathrm{Jy} / \mathrm{beam}$. The background is the $\mathrm{H} \alpha$ image from Hoopes et al. (1996).

(Beck et al. 2005). Gas and dust usually accumulates in the downstream region of bars (Athanassoula 1992). Sorai et al. (2000) indeed find that the molecular gas in NGC 253 is located in the downstream region, at $\mathrm{PA}=66^{\circ} \pm 1^{\circ}$, so that it is located between the stellar and the radio bar. The inner part of the CO-bar of about $1^{\prime}(1.1 \mathrm{kpc})$ length is also visible in the map by Sakamoto et al. (2006, Fig. 5).

The concentration of gas and dust may be due to a shock in the bar's potential that also compresses the magnetic field. Compression likely plays a role at least in the eastern bar because there the polarization degree is $22 \pm 7 \%$ (at $\lambda 3 \mathrm{~cm}$ ), a high value for a location near the midplane. The sequence from downstream to upstream is therefore regular magnetic fields, turbulent magnetic field, molecular gas, and stars. This is the reversed order that was found in the arms of spiral galaxies, as in M 51 by Patrikeev et al. (2006), so it warrants further investigation.

\subsection{Filamentary emission}

Other distinctive morphological features are the two horn-like structures that are sticking out of the central region roughly perpendicular to the direction of the bar, each consisting of two filaments ${ }^{4}$. The northern one can be clearly seen in the $\lambda 3 \mathrm{~cm}$ map that we present in Fig. 3 with a length of $30^{\prime \prime}=600$ pc. Both structures are marked in Fig. 5 as F1 and F2 north and as F3 and F4 south of the galactic plane. They are emerging from the central region that contains the nucleus, which is a strong total power source. The nuclear region is extended in the direction of the bar and has a length of $1200 \mathrm{pc}$ at this resolution. The nuclear source itself is not resolved at 7'.5 (150 pc) angular resolution. In the optical regime the morphology is dominated by absorption owing to dust filaments as shown in the high-resolution HST image from the ANGST survey (Dalcanton et al. 2007, Fig. 6).

The horn-like structures are also visible in the $120 \mathrm{~cm} \mathrm{A-}$ configuration map at a high resolution of 2 '. $2 \times 1$.' 3 , shown in Fig. 4 as an overlay on X-ray emission from Chandra data

\footnotetext{
${ }^{4}$ We note that the observed filaments are apparent structures observed in projection. They will be explained later as the walls of the nuclear outflow cones.
}

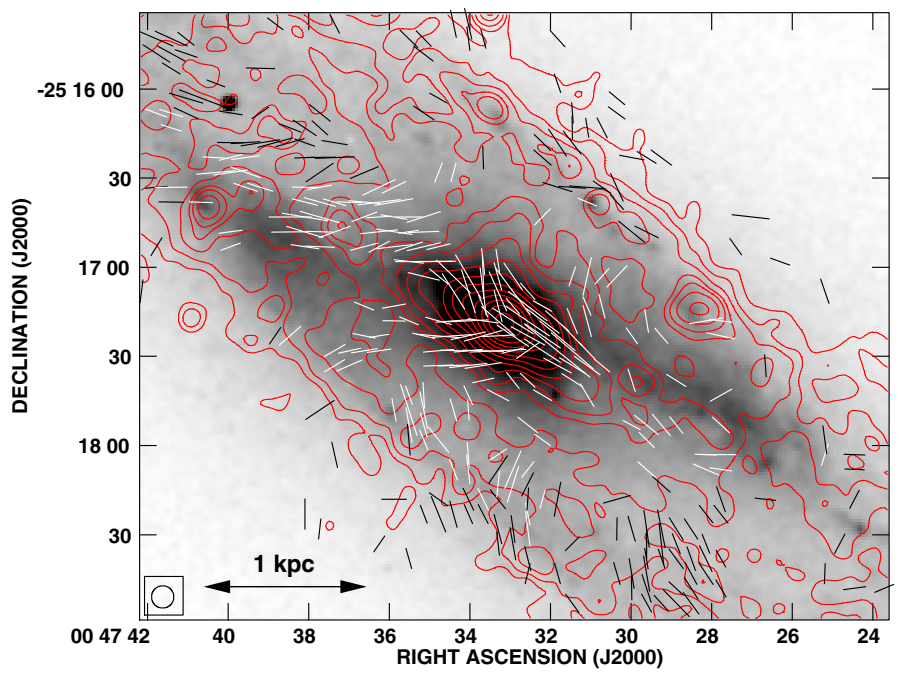

Fig. 2. Total power radio continuum at $\lambda 6 \mathrm{~cm}$ with $7 . .5$ resolution. Contours are at 5, 7, 10, 15, 20, 30, 50, 100, 200, 400, 800, 1600, 3200, and $6400 \times 100 \mu \mathrm{Jy} / \mathrm{beam}$. The vectors show the orientation of the magnetic field (not corrected for Faraday rotation) where a vector length of $1^{\prime \prime}$ is equivalent to $12.5 \mu \mathrm{Jy} /$ beam of polarized intensity. The background is a $K$ band image from the 2MASS survey (Jarrett et al. 2003).

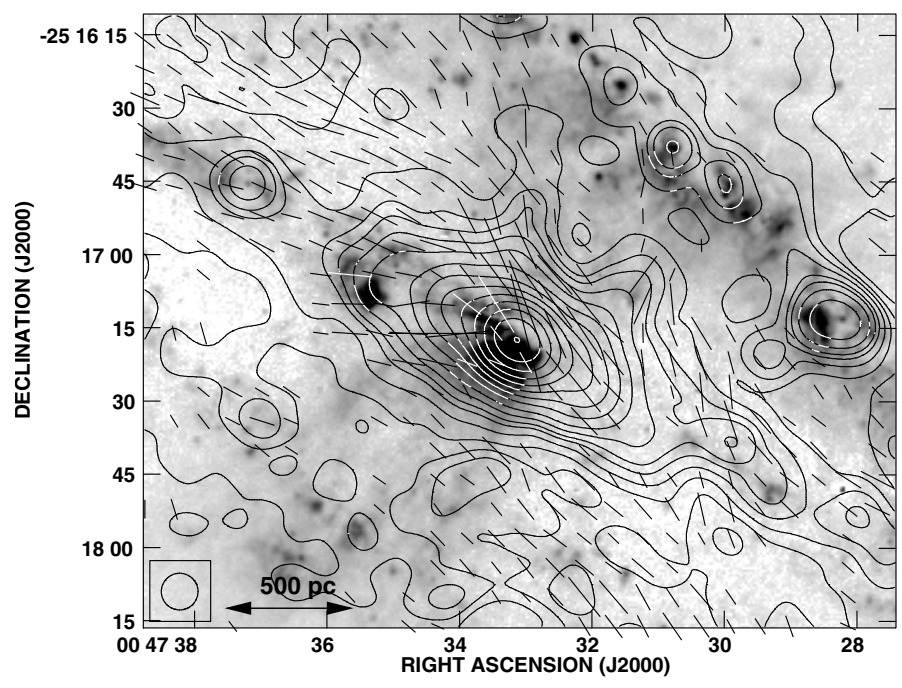

Fig. 3. Total power radio continuum at $\lambda 3 \mathrm{~cm}$ at 7.5 resolution. Contours are at $3,5,7,10,15,20,30,50,100,200,400,800,1600$, 3200 , and $6400 \times 50 \mu \mathrm{Jy} /$ beam. Vectors show the orientation of the magnetic field (assuming negligible Faraday rotation) where 1" vector length is equivalent to $12.5 \mu \mathrm{Jy}$ beam $^{-1}$ polarized intensity. The background is the $\mathrm{H} \alpha$ image from Westmoquette et al. (2011), which was scaled with a square-root function to enhance weak emission.

(Hardcastle, priv. com.). At this high resolution the nucleus (RA $00^{\mathrm{h}} 47^{\mathrm{m}} 33^{\mathrm{s}} .12$, Dec $-25^{\circ} 17^{\prime} 17^{\prime \prime} \cdot 3$ ) is resolved. The nuclear region now shows up as a structure of $900 \times 600 \mathrm{pc}$ in extent, with the longer extension aligned with the molecular bar, rather than with the major axis. Noteworthy is the apparent anticorrelation between the X-ray and the radio continuum emission in locations where the X-ray emission traces an outflow of hot gas. This is apparent in the thin X-ray filament (in green, RA $00^{\mathrm{h}} 47^{\mathrm{m}} 33^{\mathrm{s}} .2$, Dec $-25^{\circ} 17^{\prime} 10^{\prime \prime}$ ) pointing to the $\mathrm{N}$ and in the SE outflow cone, where the radio contours bend slightly inwards. The decrease in scaleheight indicates enhanced synchrotron loss due to the strong magnetic field in the outflow cone (see Paper II for details). 


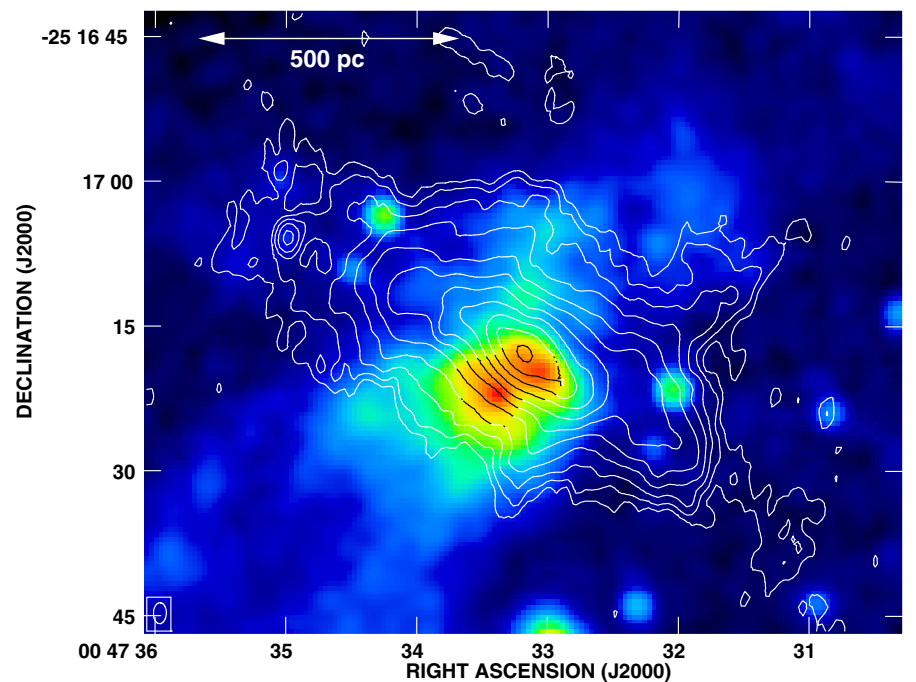

Fig. 4. Total power radio continuum at $\lambda 20 \mathrm{~cm}$ with 1 '." $3 \times 2$ '.' 2 resolution. Contours are at $-6,-3,3,6,12,25,50,100,200,400,800,1600$, and $3200 \times 50 \mu \mathrm{Jy} \mathrm{beam}^{-1}$. The background is a map of the X-ray emission between 0.5 and $5 \mathrm{keV}$ measured with the Chandra satellite convolved with a Gaussian to $2^{\prime \prime}$ resolution (Hardcastle, priv. comm.).

The filamentary structures are better visible in the greyscale map presented in Fig. 5, where the contour lines show ${ }^{12} \mathrm{CO} J=2-1$ emission observed with the Submillimeter Array (Sakamoto et al. 2006). There are four filaments that all have widths of about one beam diameter $(40 \mathrm{pc})$, so that their true width is smaller and have a length of up to $400 \mathrm{pc}$. The filaments are not exactly perpendicular to the extension but are slightly inclined, forming rather the boundary of a cone-like structure. The position angle of the filaments are $-53^{\circ} \pm 1^{\circ}(\mathrm{F} 1),-26^{\circ} \pm 1^{\circ}(\mathrm{F} 2)$, $141^{\circ} \pm 2^{\circ}(\mathrm{F} 3)$, and $116^{\circ} \pm 4^{\circ}(\mathrm{F} 4)$. The opening angles of the cones (between F1 and F2) are therefore $27^{\circ} \pm 1^{\circ}$ in the NW and between $\mathrm{F} 3$ and $\mathrm{F} 425^{\circ} \pm 4^{\circ}$ in the SE, respectively.

\subsection{Outflow cones}

The nuclear outflow of the starburst galaxy is visible as plumes of $\mathrm{H} \alpha$ and soft X-ray emission. In the NW on the far side of the halo, the emission is heavily absorbed, so that the outflow is much less visible at these wavelengths. The $\mathrm{H} \alpha$ filaments bordering the SE outflow cone coincide in shape and position with the radio filaments F3 and F4. The NW outflow cone is seen in $\mathrm{H} \alpha$ only as a weak triangular shape, where the border again coincides with the radio filaments, here F1 and F2. In Fig. 3 the $\mathrm{H} \alpha$ mission can be understood as limb brightening of the cone (see also Fig. 2 in Westmoquette et al. 2011). The $\mathrm{H} \alpha$ emission is presumably material around the rim of the cone, which cooled from the hot gas in the inner part of the cone with a temperature of $10^{7} \mathrm{~K}$. The filaments F1 and F2 in the NW are not visible in $\mathrm{H} \alpha$, probably due to absorption. The soft X-ray emission also shows some filamentary emission at the boundary of the bubbles interpreted as cooled material (Strickland et al. 2000). Using more sensitive XMM observations albeit with coarse resolution, Bauer et al. (2007) report detecting the actually hot plasma of the outflow.

By comparing the radio contour lines in Fig. 4 with the $\mathrm{CO}$ contour lines in Fig. 5, we can see several similarities. The radio filaments agree in position with extensions in $\mathrm{CO}$ emission except at the weak filament F4 that is not prominent in $\mathrm{CO}$. The minimum $\mathrm{M} 1$ on the northern side of the radio is also

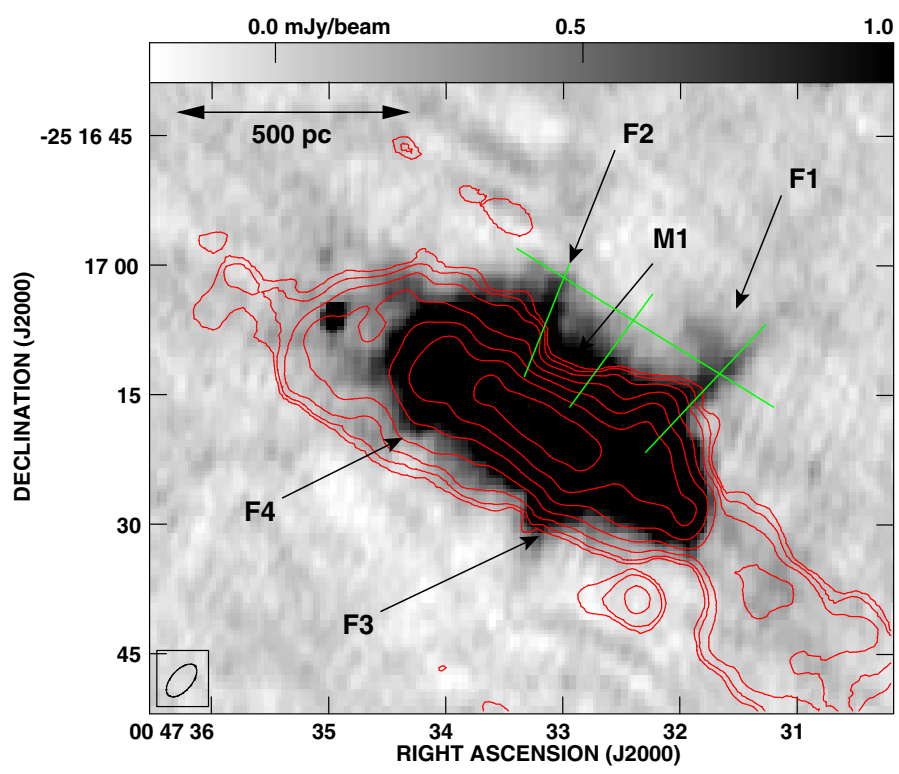

Fig. 5. ${ }^{12} \mathrm{CO} \quad J=2-1$ emission from observations with the Submillimeter Array at 4". $6 \times 22^{\prime \prime} 4$ resolution $(\mathrm{PA}=-41.2)$ from Sakamoto et al. (2006). Contours are at 3, 6, 12, 25, 50, 100, and $200 \times 7 \mathrm{Jy} \mathrm{beam}^{-1} \mathrm{~km} \mathrm{~s}^{-1}$. The background image is the $\lambda 20 \mathrm{~cm}$ total power emission with 1 '. $3 \times 2$ '. 2 resolution as shown in Fig. 4 , which shows the weak emission. The green lines show the location of the profiles discussed in Sect. 3.4. The four filaments F1-4 and the minimum M1 are denoted.

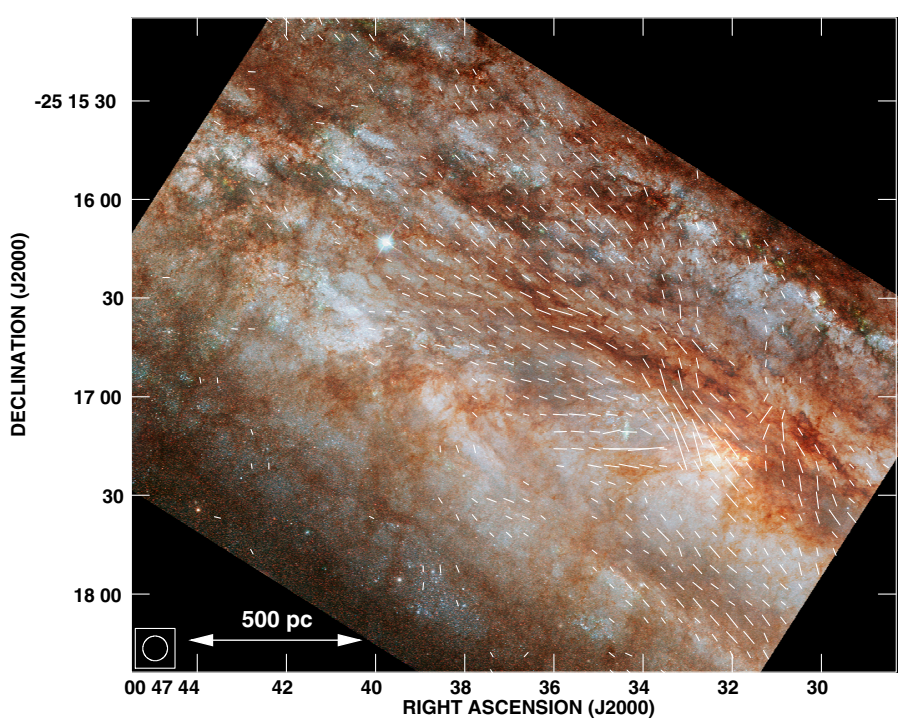

Fig. 6. Magnetic field orientation at $\lambda 3 \mathrm{~cm}$ with $7{ }^{\prime \prime} 5$ resolution overlaid on an HST image from the ANGST survey (Dalcanton et al. 2007). A vector length of $1^{\prime \prime}$ is equivalent to a polarized intensity of $21.3 \mu \mathrm{Jy}_{\text {beam }}{ }^{-1}$. The image shows only the centre and the NE part.

pronounced in CO. The similarities in the contour lines hold also for the shape for part of the nuclear region. The obvious exceptions are extensions in $\mathrm{CO}$ east and southwest of the nuclear region. We will attempt a more quantitative comparison between the radio and CO emission in Sect. 3.6.

\subsection{Emission profiles}

A useful way to characterize the distribution of the continuum emission is to measure scaleheights. We created profiles of the radio continuum emission with a fixed resolution of 

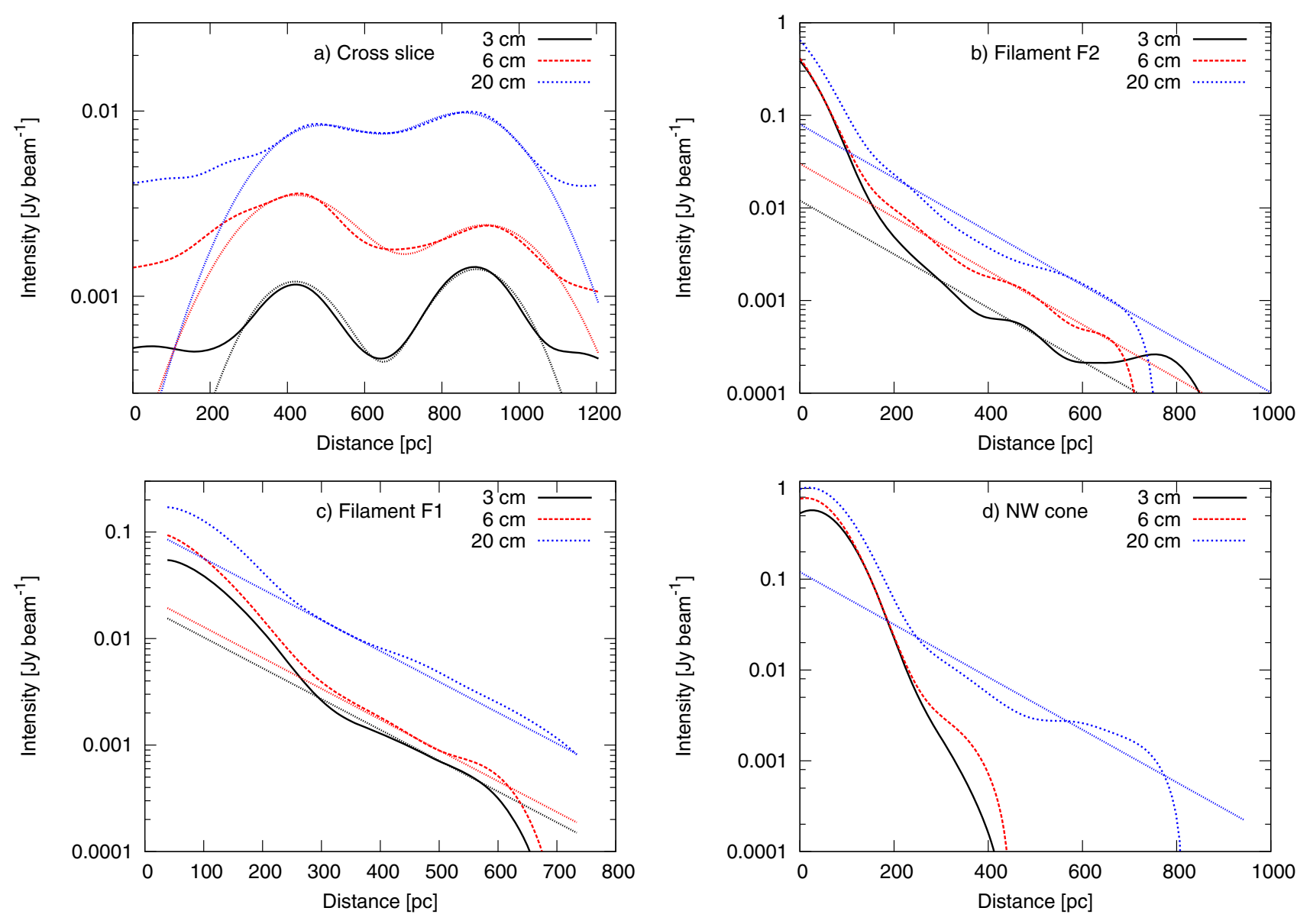

Fig. 7. Slices of continuum emission where the dotted lines show the measured profiles and the solid lines the fit to the data. a) Slice across the filaments parallel to the central region at a distance of $300 \mathrm{pc}$. b) and c) Slices along the filaments F2 and F1, respectively. d) Slice along the NW outflow cone. For slices b)-d) a constant level of background emission estimated from a) was subtracted.

7'.5 = $150 \mathrm{pc}$ for the filaments F1 and F2. The profiles, along with exponential fits, are presented in Fig. 7, where we used a slice width of $2^{\prime \prime}$, which is smaller than the resolution. Also, we used a perpendicular profile across the filaments, which shows the filaments and the depression between them. For the slices along the filaments and the cone (Figs. 7b-d), we subtracted a constant level of background emission estimated from the cross slice (Fig. 7a). The contrast between the filaments and the depression depends on wavelength. The depression is most prominent at $\lambda 3 \mathrm{~cm}$, with the level of emission in the outflow cone decreasing to the surrounding level. Therefore, at $\lambda 3 \mathrm{~cm}$ we do not detect any continuum emission coming from the interior of the outflow cone. At $\lambda 6 \mathrm{~cm}$ the intensity inside the outflow cone is not entirely decreasing to the surrounding level and at $\lambda 20 \mathrm{~cm}$ the depression is only shallow. See also Figs. 1-3, where the appearance of the filaments clearly depends on wavelength.

We fitted exponential functions to the emission profiles to measure the scaleheights. We found a scaleheight of $150 \pm 20 \mathrm{pc}$ in both filaments at all three wavelengths as shown in Figs. 7b and c. This is remarkable because the scaleheights of the continuum emission in the halo increase with increasing wavelength (Paper II). This is understood by the increase in the lifetime of cosmic-ray electrons with increasing wavelength. The wavelength-independent continuum scaleheight of the filaments indicates that we see a decrease in the magnetic field strength, rather than that of the cosmic-ray electrons density.

We fitted Gaussian profiles to the cross slices in Fig. 7a to measure the width of the filaments as a function of wavelength.
Under the assumption that the filaments have a Gaussian profile the observed width can be expressed as $F W H M_{\mathrm{obs}}^{2}=F W H M_{\mathrm{fil}}^{2}+$ $(150 \mathrm{pc})^{2}$ to account for the limited resolution, which is comparable to the width of the filaments. We found FWHMs of $330 \pm 15 \mathrm{pc}(\lambda 20 \mathrm{~cm}), 350 \pm 15 \mathrm{pc}(\lambda 6 \mathrm{~cm})$, and $250 \pm 15 \mathrm{pc}$ $(\lambda 3 \mathrm{~cm})$. We note that these values are considerably higher than the filament width of $<40 \mathrm{pc}$ that we inferred from the $\lambda 20 \mathrm{~cm}$ high-resolution map (Sect. 3.2). This result suggests a weak, extended radio emission component associated with the filaments that is not detected in the high-resolution map with a reduced sensitivity to extended emission. Both the magnetic field and cosmic-ray electrons can contribute to this weak component.

The frequency dependence of the filament width suggests a transport effect of the cosmic rays (see Sect. 5.1). A wavelengthdependent filament width is also found in supernova remnants, where the synchrotron emission in the X-ray regime exhibits extremely thin filaments $(<0.1 \mathrm{pc})$ with strong magnetic fields, whereas the radio filaments are much broader as the result of the longer lifetime and diffusion of the lower energy electrons (Reynolds et al. 2011).

\subsection{Radio spectral index}

The emission profiles with the same scaleheight in the filaments imply that the radio spectral index $\alpha$ (defined as $S \propto v^{\alpha}$ ) is constant with respect to the distance from the disc. To calculate the radio spectral index, we took the flux density from the fits 


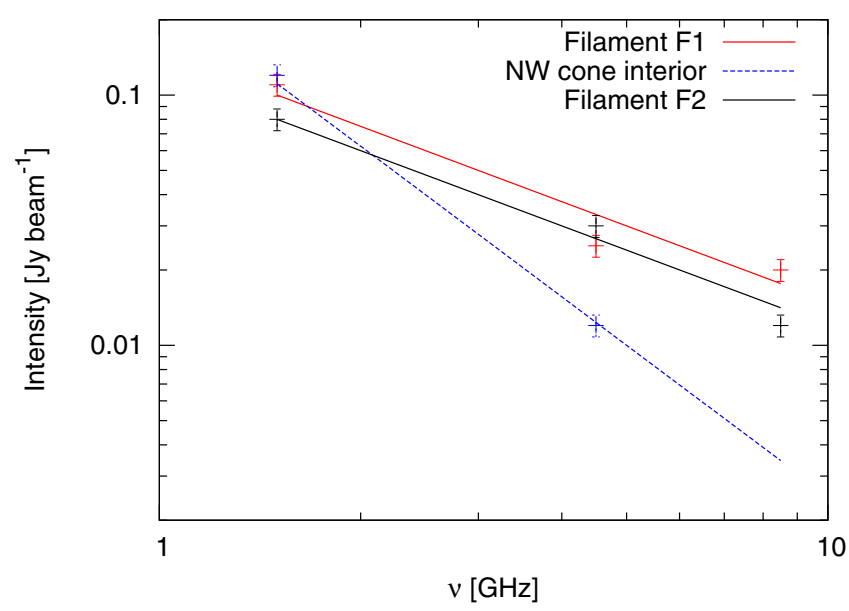

Fig. 8. Spectra of the radio continuum flux density as measured from the fits to the data shown in Fig. 7.

shown in Fig. 7 extrapolated to a distance of 0 pc. For both filaments we find $\alpha=-1.0 \pm 0.1$, with no significant curvature in the spectra shown in Fig. 8. In filament F2 the $\lambda 3 \mathrm{~cm}$ flux density is low hinting at strong electron losses. In contrast, the $\lambda 3 \mathrm{~cm}$ flux density is high in filament F1 where the thermal fraction may be greater. Of course, with only three different wavelengths, we cannot measure any curvature precisely but can assume a linear spectrum. The radio spectra in the filaments are consistent with a non-thermal spectrum within the error interval, with a nonthermal radio spectral index of $\alpha_{\mathrm{nt}}=-1$. In the interior of the cone (see Fig. $7 \mathrm{a}$ ), the radio spectral index is $-2.0 \pm 0.2$ between $\lambda \lambda 20$ and $6 \mathrm{~cm}$, indicating strong energy losses of the electrons.

In Fig. 9 we present the distribution of the radio spectral index between $\lambda \lambda 20$ and $3 \mathrm{~cm}$. We clipped each map below $4 \times$ the rms noise level prior to the combination, so that the maximum error is \pm 0.3 in the radio spectral index. The spectrum is flat in the centre around the nucleus but steepens significantly away from it. The two filaments in the NW and the radio bar show up as extensions of the flat central region. The flat spectral index of the central region also extends into the radio bar on both sides of the nuclear region. At the location of the NW outflow cone (RA $00^{\mathrm{h}} 47^{\mathrm{m}} 32^{\mathrm{s}}$, Dec $-25^{\circ} 16^{\prime} 50^{\prime \prime}$ ), the radio spectral index steepens significantly, as expected for strong radiation losses of the electrons (Sect. 5.1). We checked carefully that this feature is not caused by the merging of the interferometric data with the single-dish data. It is already apparent in the radio spectral index map constructed from VLA data alone. The steep region south of the nucleus (RA $00^{\mathrm{h}} 47^{\mathrm{m}} 33^{\mathrm{s}}$, Dec $-25^{\circ} 17^{\prime} 50^{\prime \prime}$ ), however, is less certain. At $\lambda 3 \mathrm{~cm}$ we observe a minimum in the total power emission that may be caused by the high dynamic range close to the nucleus. Also the position does not not agree with the SE outflow cone (see Fig. 3), so that this feature could be an artefact.

\subsection{Radio continuum as star formation tracer}

The radio continuum emission is a star formation tracer because it is sensitive to the thermal and the non-thermal emission. UVphotons emitted by massive stars ionize the hydrogen that can be seen as thermal $\mathrm{H} \alpha$ and free-free radio emission. Supernova remnants that mark the end of life of massive stars accelerate cosmic-ray electrons that radiate synchrotron emission, the non-thermal component of the radio emission. Condon (1992)

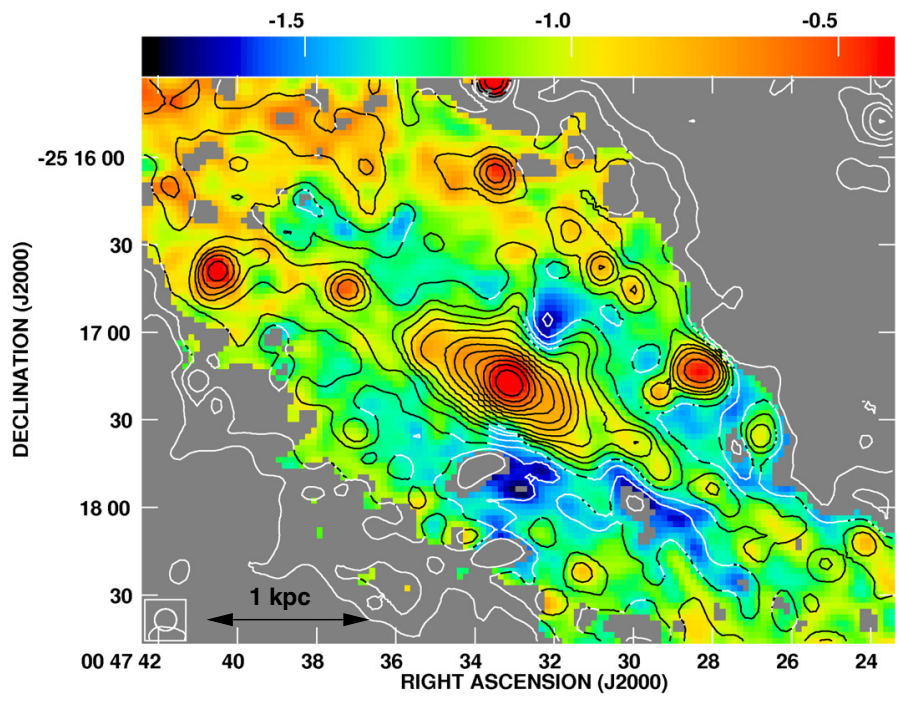

Fig. 9. Radio spectral index between $\lambda \lambda 20$ and $3 \mathrm{~cm}$ at 7 '. 5 resolution as grey-scale. Contours show the continuum emission at $\lambda 3 \mathrm{~cm}$ as in Fig. 3.

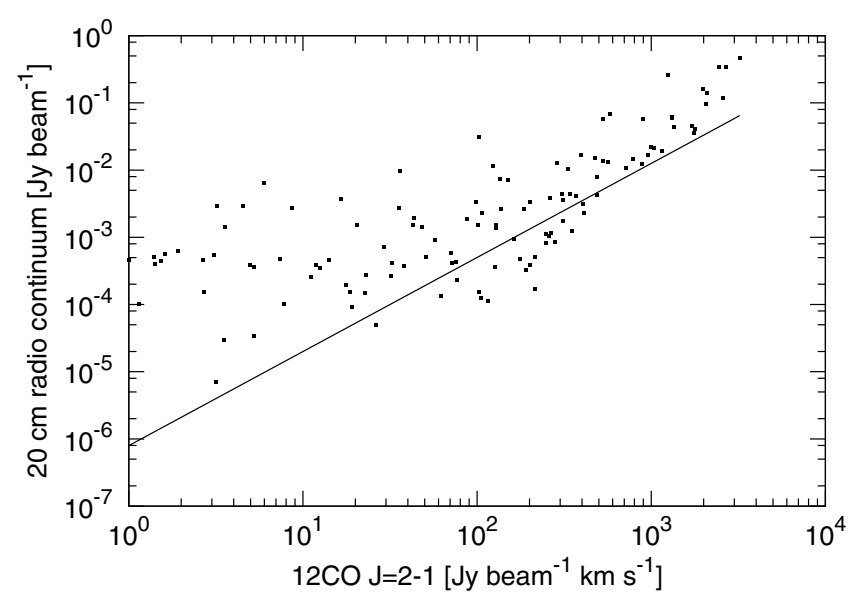

Fig. 10. Pixel-by-pixel comparison of the $\lambda 20 \mathrm{~cm}$ radio continuum intensity with the ${ }^{12} \mathrm{CO} J=2-1$ intensity at $88 \mathrm{pc}$ resolution. The line shows the theoretical expectation using the conversion into star formation rates as explained in the text.

calculated the star formation rate or, for resolved emission, the star formation rate density as

$$
\left(\frac{\mathrm{SFRD}}{M_{\odot} \mathrm{yr}^{-1} \mathrm{kpc}^{-2}}\right)_{>0.1 M_{\odot}}=1.4 \times 10^{-7}\left(\frac{I_{20 \mathrm{~cm}}}{\mathrm{Jy} \mathrm{ster}^{-1}}\right),
$$

where $I_{20 \mathrm{~cm}}$ is the radio continuum intensity at $\lambda 20 \mathrm{~cm}$.

Another star formation tracer is the molecular hydrogen traced by the ${ }^{12} \mathrm{CO} J=2-1$ emission. According to Kennicutt (1998) the star formation rate density relates to the mass surface density of combined atomic and molecular hydrogen $\Sigma_{\mathrm{H} 2}$ as

$$
\left(\frac{\mathrm{SFRD}}{M_{\odot} \mathrm{yr}^{-1} \mathrm{kpc}^{-2}}\right)=2.5 \times 10^{-10}\left(\frac{\Sigma_{\mathrm{gas}}}{M_{\odot} \mathrm{yr}^{-1} \mathrm{kpc}^{-2}}\right)^{1.4}
$$

We assume that the entire gas in the nuclear region is of molecular nature and estimate the gas mass thus from the molecular hydrogen alone, which we derived from the $\mathrm{CO}$ map. We adopted a ratio between ${ }^{12} \mathrm{CO}(2-1)$ and ${ }^{12} \mathrm{CO}(1-0)$ of 0.9 found by Weiß et al. (2005) for the starburst galaxy M 82. Also we used the 


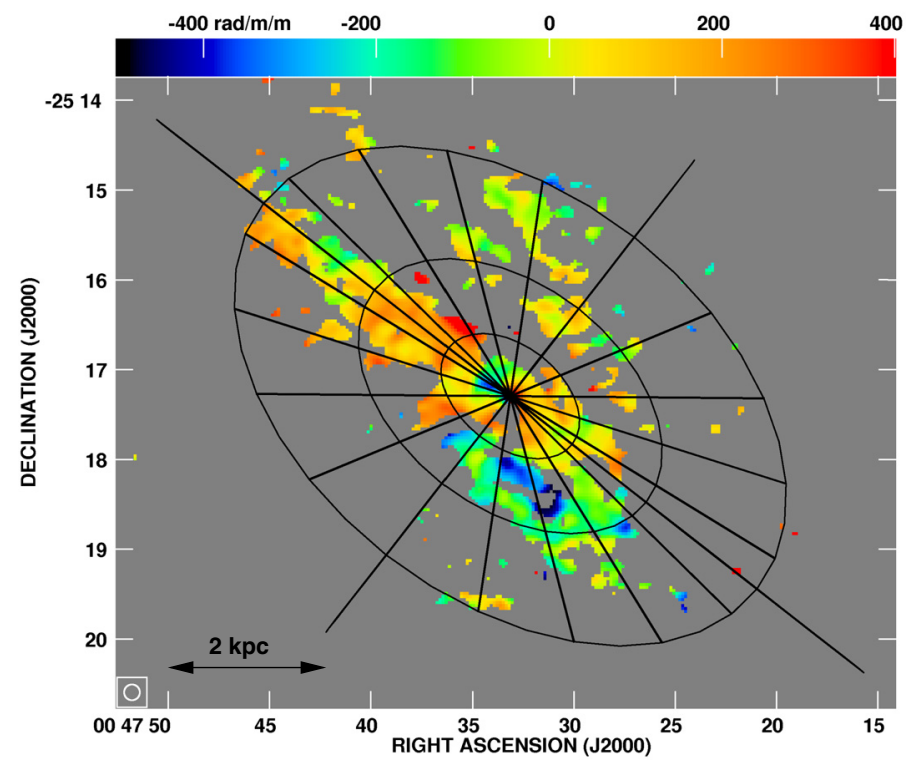

Fig. 11. Rotation measure distribution between $\lambda \lambda 6$ and $3 \mathrm{~cm}$ with a resolution of 10'.5. The major and minor axes and the position of the sectors are shown.

conversion of $5 \times 10^{19}$ molecules $\mathrm{cm}^{-2} /\left(\mathrm{K} \mathrm{km} \mathrm{s}^{-1}\right)$ to convert from $\mathrm{CO}$ to $\mathrm{H}_{2}$ column density, which was derived by Downes \& Solomon (1998) for ultraluminous far-infrared (FIR) galaxies and is widely used for starburst galaxies in the literature.

In Fig. 10 we compare the radio continuum intensity at $\lambda 20 \mathrm{~cm}$ and the ${ }^{12} \mathrm{CO} J=2-1$ intensity on a scale of $4 . \prime 6=88 \mathrm{pc}$ in a pixel-by-pixel plot. The line shows the relation, if we use the conversion into star-formation rate densities. The scatter in the data points is large, with the radio flux densities on average a factor of 4 brighter than theoretically expected. Ott et al. (2005) find that the integrated star formation rate in the nucleus of NGC 253 as derived from $\lambda 1.2 \mathrm{~cm}$ radio continuum observations agrees with that of the FIR, suggesting that the radio emission is a reliable tracer for star formation. Recent studies established such a correlation in spatially resolved observations. Dumas et al. (2011) confirm a tight correlation between radio emission and various other star formation tracers down to a scale of $240 \mathrm{pc}$. We do not find such a tight correlation in the nucleus of NGC 253. A possible explanation are transport effects of the cosmic rays in the powerful nuclear outflow, which we discuss in Sects. 5.1-5.3. If cosmic rays diffuse away from the star formation sites, the radio emission gets decoupled from other star formation tracers, and the relation between star formation rate and radio emission breaks down.

\section{Magnetic field structure}

\subsection{Magnetic field orientation}

In Figs. 3 and 6 we show the magnetic field orientation at $\lambda 3 \mathrm{~cm}$, where the length of the vectors is proportional to the polarized intensity. The maximum polarized intensity is near the nucleus, in the adjacent NE region. The distribution is asymmetric with respect to the minor axis, the NE half having a higher fraction of polarized intensity. There is a local minimum in the NW outflow cone where we do not detect polarized emission, coincident with the minimum in total power. The magnetic field orientation is parallel to the major axis only NE of the nucleus near the stellar bar. In all other regions there is a significant vertical component particularly in the two radio filaments bordering the NW outflow

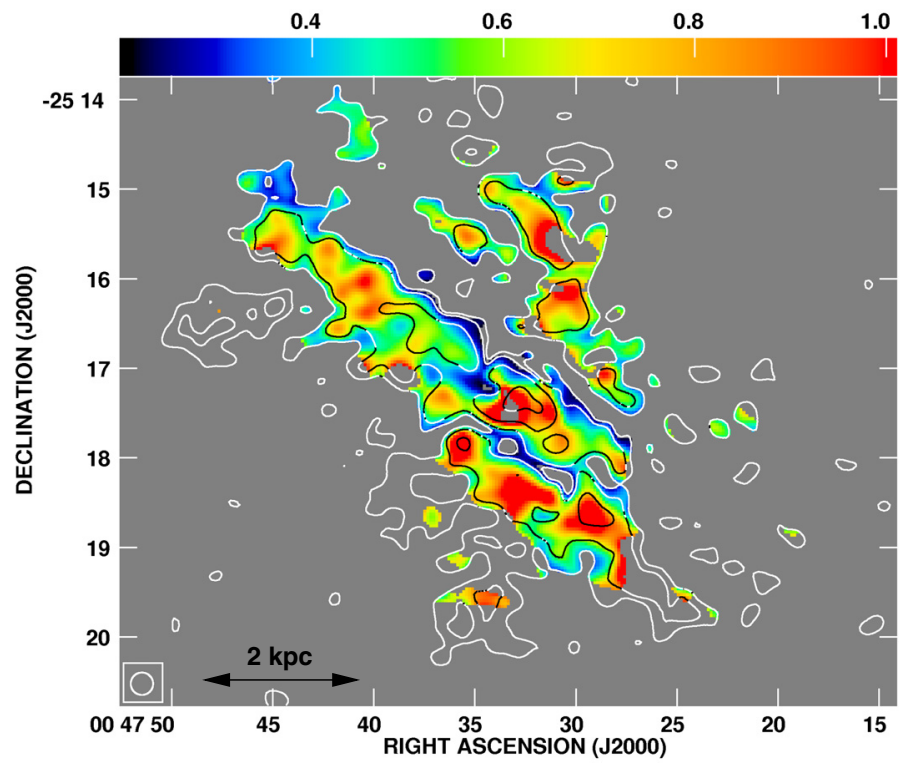

Fig. 12. Distribution of the depolarization between $\lambda \lambda 6$ and $3 \mathrm{~cm}$ at a resolution of $15^{\prime \prime}$. Contours show the distribution of the polarized emission at $\lambda 6 \mathrm{~cm}$ with contours at $3,5,10$, and $20 \times 70 \mu \mathrm{Jy}_{\text {beam }}{ }^{-1}$, the rms noise level.

cone. There, the magnetic field is also remarkably well aligned with a polarization degree of $15 \pm 5 \%$.

For the study of the Faraday rotation we made a map in polarization at $\lambda 6 \mathrm{~cm}$ (see Fig. 2). Because the integration time is short (30 min), the map is not as sensitive as the $\lambda 3 \mathrm{~cm}$ map. The polarized emission is again asymmetric with respect to the minor axis with most of the emission in the NE and is aligned roughly along the major axis. The magnetic field orientation is different in places from that at $\lambda 3 \mathrm{~cm}$ indicating strong Faraday rotation, which we discuss in the next section.

\subsection{Rotation measure distribution}

The rotation measure (RM) is given by the line-of-sight component of the large-scale magnetic field. If the RM is positive the magnetic field is pointing towards us and if it is negative it points away from us. In Fig. 11 we present the RM distribution between $\lambda \lambda 6$ and $3 \mathrm{~cm}$ at 10 .' 5 resolution. The maps of the polarization angle were clipped below a polarized intensity of $3 \times$ the r.m.s. noise level, prior to the combination. The RM ambiguity is $\approx \pm n \cdot 1200 \mathrm{rad} \mathrm{m}^{-2}$.

The RM changes on scales of a few beam diameters, corresponding to about $100 \mathrm{pc}$. This finding agrees with the observations in face-on galaxies, which show similar RM distributions, e.g. in M 51 (Fletcher et al. 2011). There the halo RM is also expected to contribute to the RM signal as a foreground to the bright disc. Such frequent RM reversals can hardly be observed in edge-on galaxies due to the effect of averaging along the lineof-sight. The RM fluctuations may be caused by field reversals on small scales, possibly an "anisotropic" field, which is generated from an isotropic turbulent field by compression or shear ${ }^{5}$.

\footnotetext{
5 The observed B-vectors of linearly polarized emission can trace either regular magnetic fields (i.e. preserving their direction within the telescope beam) or anisotropic fields (i.e. with multiple field reversals within the beam). Anisotropic fields can be generated from turbulent fields by shear or compression. To distinguish between these two components, additional Faraday rotation data is needed. The fields observed in polarization are called "ordered" throughout this paper.
} 

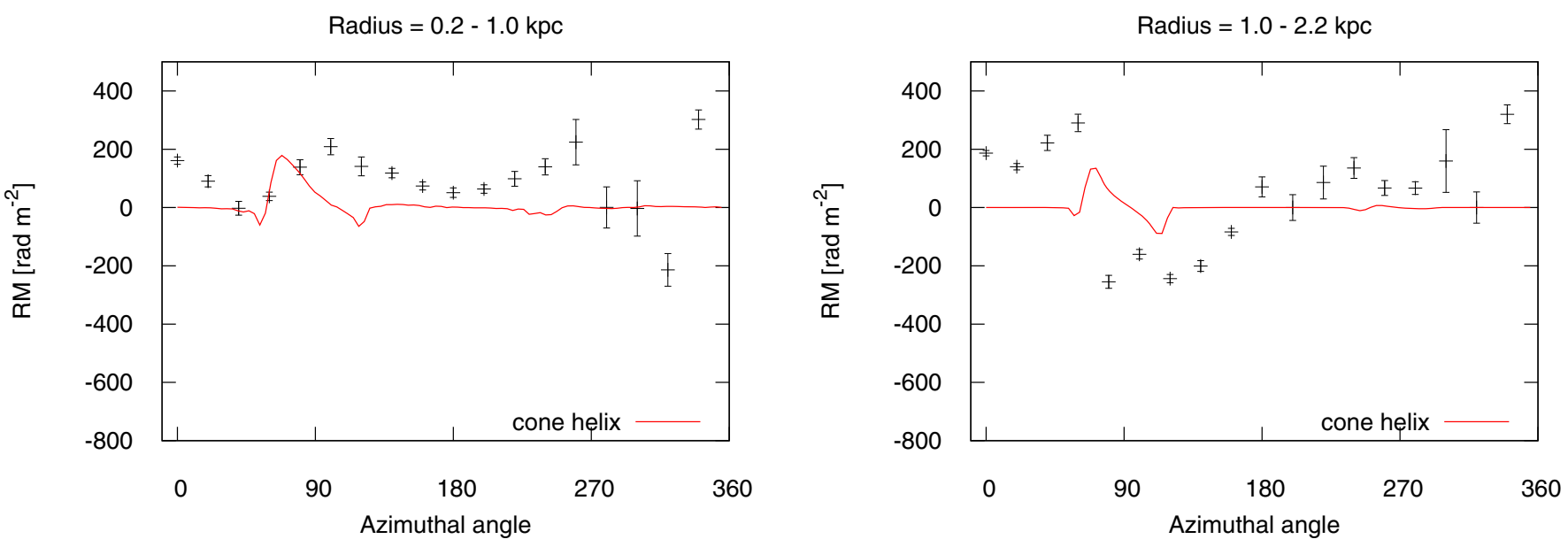

Fig. 13. RMs averaged in azimuthal sectors in the inner disc. The red line shows the model of the helical magnetic field in the outflow cone as described in the text (no fit).

The RM is typically between -200 and $+200 \mathrm{rad} \mathrm{m}^{-2}$, far away from any RM ambiguity between $\lambda \lambda 6$ and $3 \mathrm{~cm}$, so the orientation of the magnetic field vectors at $\lambda 3 \mathrm{~cm}$ are rotated by at most $\pm 15^{\circ}$. The rotation angle is small, so that we do not apply any correction to the polarization angles at this wavelength. We note that the galactic foreground RM for NGC $253\left(l=97^{\circ}\right.$, $b=-88^{\circ}$ ) is only a small contribution at $10 \pm 5 \mathrm{rad} \mathrm{m}^{-2}$ (Noutsos et al. 2008; Taylor et al. 2009), which we neglect in the further analysis.

\subsection{Faraday depolarization}

The observed degree of polarization is usually lower than the theoretically highest value of about $74 \%$. Relevant depolarization may be caused by wavelength-independent beam depolarization and by wavelength-dependent depolarization effects, the Faraday depolarization. The latter consists of differential Faraday rotation (related to the regular magnetic field strength and thermal electron density within the emitting source, the galaxy), of Faraday dispersion related to the turbulent magnetic field strength within the emitting source (internal Faraday dispersion), and of a turbulent magnetic field between the galaxy and the observer (external Faraday dispersion) (Burn 1966; Sokoloff et al. 1998). Usually, the differential Faraday rotation is expected to contribute most to the observed depolarization within a spiral galaxy, because its values are related to the observed RMs within this galaxy.

Observationally, the Faraday depolarization is calculated as the ratio between the observed degrees of polarization in maps at two different wavelengths smoothed to the same angular resolution or directly by DP $=\left(\mathrm{PI}_{1} / \mathrm{PI}_{2}\right) \times\left(v_{2} / v_{1}\right)^{\alpha_{\mathrm{nt}}}$, where $\alpha_{\mathrm{nt}}$ is the non-thermal spectral index. Using $\alpha_{\mathrm{nt}}=-1$ as measured Paper I, we calculated DP between $\lambda \lambda 6$ and $3 \mathrm{~cm}$ at $15^{\prime \prime}$ resolution presented in Fig. 12.

The value of DP is for most parts in the galaxy is rather low with values between 0.6 and 1.0 where $|R M|$ reaches values up to $250 \mathrm{rad} \mathrm{m}^{-2}$. As a result, the expected depolarization at $\lambda 6 \mathrm{~cm}$ due to differential Faraday rotation alone leads to $0.65 \leq \mathrm{DP} \leq$ 1.0 and indicates that other depolarization effects are negligible. The depolarization increases with higher distances $\mathrm{N}$ of the major axis up to values of about RM $=350 \mathrm{rad} \mathrm{m}^{-2}$ where the differential depolarization leads to DP $=0.2$, followed by a stripe with no polarized emission at $\lambda 6 \mathrm{~cm}$ parallel to the major axis but offset in a NW direction. This stripe has an almost linear, well-defined boundary, and is probably caused by even stronger Faraday depolarization of the regular and turbulent magnetic field in the disc that lies in front.

More to the NW, we again detect polarized emission at $\lambda 6 \mathrm{~cm}$ where DP again lies within 0.6 and 1.0. We conclude that if we restrict the study of RM to the near side (SE of the major axis), our analysis should hardly be affected by depolarization.

\subsection{Disc magnetic field}

According to the mean-field dynamo theory in thin-disc objects, the axisymmetric spiral (ASS) mode $(m=0)$ with even symmetry is the dominant pattern of the regular field (Beck et al. 1996). Our azimuthal RM analysis in Paper II showed a single periodical variation, in accordance with an $m=0$ mode. Our new observations allowed us to test this in more detail and to calculate refined ASS models. We compared the models with the observations using averaging in sectors to plot the azimuthal RM variation. The radial interval should avoid the starburst region and separate the molecular bar region from the disc. The nuclear starburst region extends to a radius of $\approx 300 \mathrm{pc}$. The molecular bar extends to a galactocentric radius of $3.5 \mathrm{kpc}$ (Sorai et al. 2000), while the bright part extends to only about 1 kpc (Fig. 5). We chose $2.2 \mathrm{kpc}$ as a compromise. As the outer radius we take $4.0 \mathrm{kpc}$, up to where the signal-to-noise ratio allows us the integration. In summary, we used three radius intervals, $0.2-1.0 \mathrm{kpc}$, $1.0-2.2 \mathrm{kpc}$ and $2.2-4.0 \mathrm{kpc}$, where we chose an inclination angle of $50^{\circ}$ and an azimuthal spacing of $20^{\circ}$ (the position of the sector rings is shown in Fig. 11). For the noise estimate we used the rms noise values of Stokes $Q$ and $U$.

Figure 13 shows that, in the inner and middle ring, $|\mathrm{RM}|$ has no maxima around the major axis (azimuthal angle $0^{\circ}$ and $180^{\circ}$ ), as would be expected for an axisymmetric $m=0$ mode. Instead, $|\mathrm{RM}|$ is strongly enhanced and changes its sign near the minor axis (azimuthal angles $90^{\circ}$ and $270^{\circ}$ ) where the $m=0$ mode cannot contribute to RM. We conclude that the disc magnetic field within $\approx 2.2 \mathrm{kpc}$ does not have a regular axisymmetric structure. To explain the lack of RM near the major axis, we propose that the ordered field in the inner disc is anisotropic, i.e. it contributes to the polarized intensity but has frequent reversals, so that it does not contribute to RM. In Sect. 4.5 we discuss a model 


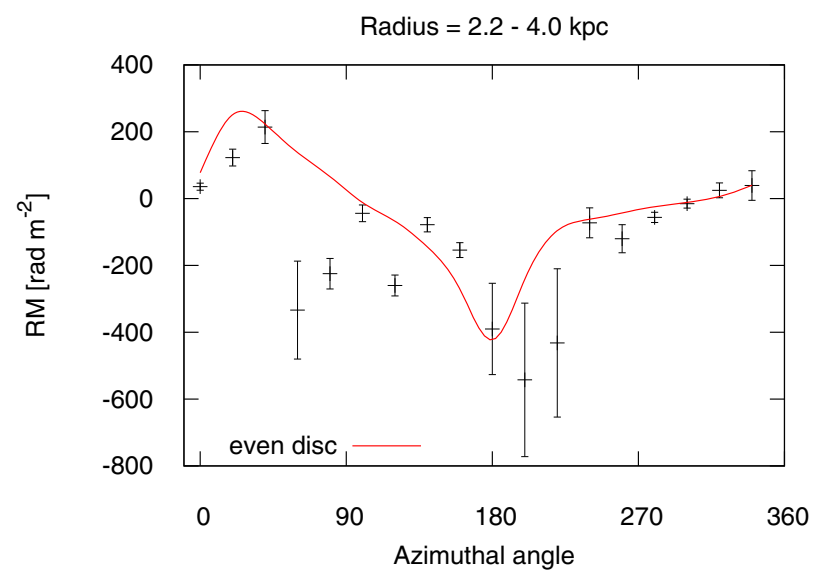

Fig. 14. Azimuthally averaged RMs in the outer disc where the red line shows an even-parity axisymmetric spiral model of the disc field.

of the conical outflow that can explain the RM enhancements within $2.2 \mathrm{kpc}$.

In the outer ring $(2.2-4.0 \mathrm{kpc})$, shown in Fig. 14, the evenparity axisymmetric disc magnetic field gives a reasonable fit to the data, except around $60^{\circ}-80^{\circ}$. This deviation can be attributed to the "radio spur" that has already been identified by Carilli et al. (1992) in total power radio continuum and which has a strong vertical field component. This finding agrees with our previous observations (Paper II), where we detected an even-parity axisymmetric disc field at lower resolution. Here we used the same model parameters as in Paper II, except that the anisotropic component in the even disc model is zero. The model is therefore no fit to the data.

\subsection{Magnetic field in the outflow cone}

In this section we test whether the magnetic field in the outflow cone can explain the RM enhancements around the minor axis seen in Fig. 13. No polarized emission from an extended halo field is detected with our observations as the magnetic field vectors are mostly parallel to the disc. The magnetic field only opens up farther away from the galactic midplane and at larger galactocentric radii (see Paper II). However, our high-resolution image (Fig. 5) suggests that the outflow cone has a strong magnetic field aligned with the walls of the cone, so can influence $\mathrm{RM}$, particularly in the SE cone that is located in front of the bright polarized disc emission and may act as a Faraday screen. We made models using an anisotropic magnetic field in the disc and a filamentary magnetic field distributed in the walls of the conical outflow. In Table 2 we summarize the parameters of the model, and we show a sketch in Fig. 15. We assumed a rather large cone opening angle of $45^{\circ}$ as the cone should open farther away from the disc.

The model RM distribution is shown in Fig. 16 along with the magnetic field orientation. The magnetic field orientation is influenced little by the magnetic field in the outflow cone because its $|\mathrm{RM}|$ of $\leq 200 \mathrm{rad} \mathrm{m}^{-2}$ corresponds to a rotation of less than $46^{\circ}$. In the NW, the outflow cone is almost not detected in $\mathrm{RM}$ as it is located behind the bright polarized disc. The vertical component of the magnetic field points away from the disc in the SE outflow cone (see Paper II). However, a vertical field alone cannot give rise to the observed strong change in RM. We also need an azimuthal component parallel to the rotation direction of the disc. Both components together form a helical
Table 2. Parameters for the synthetic polarization maps (other parameters as in Paper II).

\begin{tabular}{lcl}
\hline \hline Parameter & Value & Notes \\
\hline Filament width & $40 \mathrm{pc}$ & from $\lambda 20 \mathrm{~cm}$ \\
Base length & $300 \mathrm{pc}$ & from $\lambda 20 \mathrm{~cm}$ \\
Disc field scaleheight $h_{\mathrm{B}}^{\text {disc }}$ & $1.6 \mathrm{kpc}$ & thin radio disc \\
Disc field strength $B_{0}^{\text {disc }}$ & $4.4 \mu \mathrm{G}$ & from equipartition \\
Filament field strength $B_{0}^{\text {fil }}$ & $20 \mu \mathrm{G}$ & from equipartition \\
Electron density $n_{\mathrm{e}}$ & $2 \mathrm{~cm}^{-3}$ & from $\mathrm{H} \alpha$ and soft X-ray \\
Electron scaleheight $h_{\mathrm{e}}$ & $1400 \mathrm{pc}$ & from $\mathrm{H} \alpha$ \\
Cone opening angle $2 \beta$ & $45^{\circ}$ & \\
CRE scaleheight disc & $800 \mathrm{pc}$ & thin radio disc \\
CRE scaleheight filament & $200 \mathrm{pc}$ & filaments \\
Filament field scaleheight $h_{\mathrm{B}}^{\text {fil }}$ & $600 \mathrm{pc}$ & from equipartition \\
\hline
\end{tabular}

Notes. $\mathrm{CRE}=$ cosmic-ray electrons.

magnetic field, which generates the strongest RM signals in the walls of the outflow cone. We assumed that the azimuthal component rises linearly to a height of $1200 \mathrm{pc}$, where the azimuthal component is equal to the vertical field component (opening angle $\alpha=45^{\circ}$, see Appendix). As shown in Fig. 15, the field lines are vertical close to the disc but start to wind up with increasing height.

Our model can basically explain the observed RM distribution, especially the RM jump between $60^{\circ}$ and $80^{\circ}$ in the middle ring $(1.0-2.2 \mathrm{kpc})$. The red lines from the model shown in Fig. 13 have the right properties, but are not good fits. In the inner ring, the RM peak is at an too small azimuthal angle, probably because the cone is not oriented perfectly perpendicular to the disc. The RM amplitudes of the model are too small in the middle ring, which indicates that the azimuthal field components increases faster than linearly with radius. An improved model would need more parameters than those given in Table 2, but with the present observations we did not attempt to constrain them.

\subsection{Magnetic field strengths}

We estimated the magnetic field strength from the radio intensities by assuming equipartition between the energy densities of the magnetic field and the total cosmic rays (Beck \& Krause 2005). As we are only measuring the electrons we have to make an assumption about the proton to electron ratio, $K=100$, the usually assumed value for galaxies.

This choice of the $K$-factor is motivated by theory and observations. Firstly, if the acceleration process produces a power law in momentum of the cosmic rays and the initial numbers of protons and electrons are equal, then one can infer that for relativistic energies the $K$-factor depends only on the mass ratio of protons to electrons, as shown e.g., by Schlickeiser (2002, Sect. 19.4) and Beck \& Krause (2005, their appendix). Secondly, local measurements in the solar system can directly lead to the proton and electron spectrum and corroborate $K=100$. Adriani et al. (2011) present an electron spectrum based on the satellite experiments PAMELA. From their Fig. 1 one can infer the electron flux as $0.18 \mathrm{~s}^{-1} \mathrm{~m}^{-2} \mathrm{GeV}^{-1}$ (converting their units). Shikaze et al. (2007) present a proton spectrum based on the balloon experiment BESS. From their Fig. 7, a proton flux of $20 \mathrm{~s}^{-1} \mathrm{~m}^{-2} \mathrm{GeV}^{-1}$ is inferred. Thus, the solar system value agrees with $K=100$. 


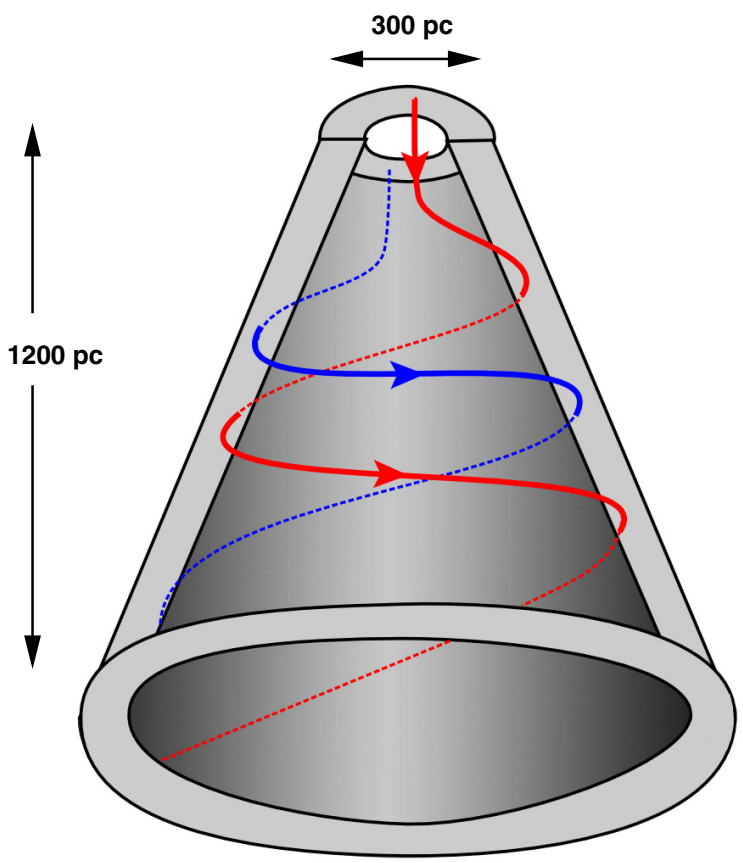

Fig. 15. Sketch of the magnetic field in the walls bordering the SE outflow cone, which appears as filaments when seen in projection. Solid lines show field lines on the front side of the cone, whereas dotted lines show them on the rear side.

The $K$-factor is nevertheless somewhat uncertain for external galaxies and thus induces uncertainties in the equipartition magnetic field strength. Particularly, $K$ may be higher in case of strong energy losses of the electrons, where the electrons have died away and only the protons (and nuclei) remain. This may be the case for galactic haloes which contain an aged population of cosmic-ray electrons. As we have seen in Sect. 3.5, the synchrotron losses in the nuclear outflow of NGC 253 are important, so that the $K$-factor may well be larger than 100 ; however the magnetic field strength depends on $K^{1 / 4}$, so that a $50 \%$ error in $K$ produces only a $13 \%$ error in the magnetic field strength. Summarizing, our magnetic field strengths should be taken as lower limits.

The radio intensities were taken at $\lambda \lambda 20,6$, and $3 \mathrm{~cm}$ from the fits to the filaments, as shown in Fig. 7, and from the maps in Figs. 1-3, where we subtracted the background emission from the disc. The thermal contribution that also needs to be subtracted (expect for the nucleus) was assumed to be $10 \%$ $(\lambda 20 \mathrm{~cm}), 20 \%(\lambda 6 \mathrm{~cm})$, and $30 \%(\lambda 3 \mathrm{~cm})$. The nuclear region has a size of $600 \mathrm{pc}$, which we took as the integration length along the line-of-sight, resulting in a magnetic field strength of $160 \pm 20 \mu \mathrm{G}$. For the region of the outflow cone we took $500 \mathrm{pc}$, which is the diameter of the outflow cone. The result is $B_{\text {tot }}=46 \pm 10 \mu \mathrm{G}$ for the total field and $B_{\text {ord }}=21 \pm 5 \mu \mathrm{G}$ for the ordered field. The intensity of the filaments F1 and F2, observed at $\lambda 20 \mathrm{~cm}$ at high resolution (Fig. 5) with a width of $<40 \mathrm{pc}$, yields a lower limit of the total field strength of $40 \mu \mathrm{G}$ and $18 \mu \mathrm{G}$ for the ordered field.

\section{Discussion}

\subsection{Cosmic-ray transport}

As found in Sect. 4.5, the helical magnetic field lines are confined to the walls that border the outflow cones, which appear as

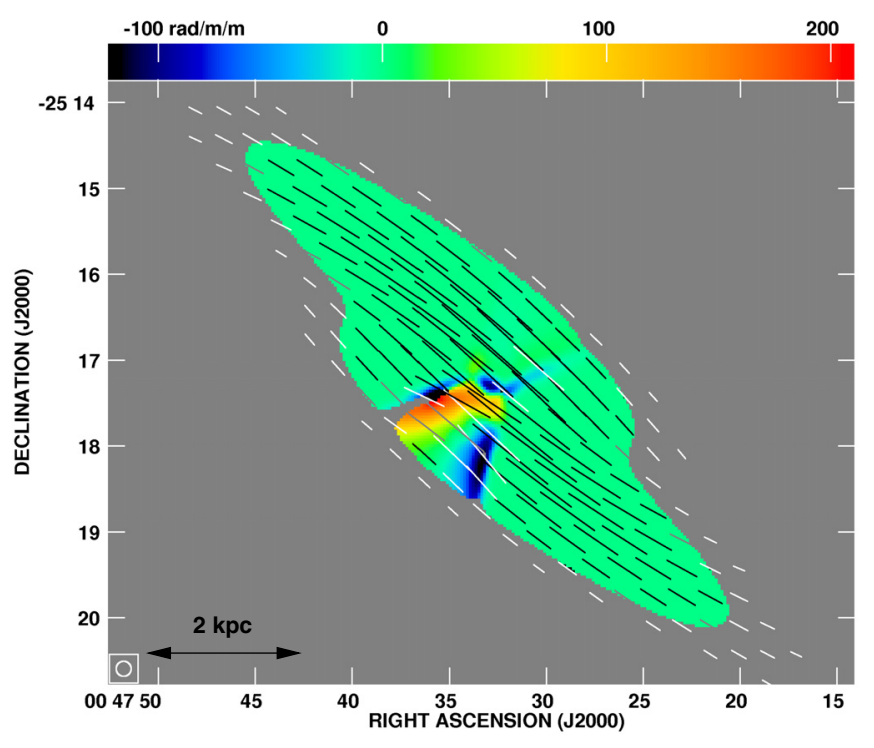

Fig. 16. Model RM distribution and magnetic field orientation at $\lambda 3 \mathrm{~cm}$ at 10.5 resolution as explained in the text.

filaments when observed in projection. Therefore, we can study the cosmic-ray transport both, parallel to the filament direction and also perpendicular to it.

Galactic cosmic-ray electrons with energies of a few $\mathrm{GeV}$ lose their energy mainly by synchrotron and inverse Compton (IC) radiation, and by adiabatic losses. Other losses like ionization ones can be neglected for the typical conditions of the interstellar medium. The conditions inside the nuclear region are different, and ionization losses may dominate (Thompson et al. 2006), but we only investigate relativistic electrons here that have escaped from the nuclear region.

The combined synchrotron and IC losses for a single cosmicray electron is given by

$\frac{\mathrm{d} E}{\mathrm{~d} t}=-\frac{4}{3} \sigma_{\mathrm{T}} c\left(\frac{E}{m_{\mathrm{e}} c^{2}}\right)^{2}\left(U_{\mathrm{ph}}+U_{\mathrm{B}}\right)$,

where $U_{\mathrm{ph}}$ is the photon energy density, $U_{\mathrm{B}}$ the magnetic field energy density, $\sigma_{\mathrm{T}}=6.65 \times 10^{-25} \mathrm{~cm}^{2}$ is the Thomson cross section, and $m_{\mathrm{e}}=511 \mathrm{keV} \mathrm{c}^{-2}$ the electron rest mass. The time dependence of the energy is $E(t)=E_{0} /\left(1+t / t_{\mathrm{e}}\right)$, where the electron lifetime is defined as the time at which the electron has lost half its initial energy

$t_{\mathrm{e}}=5.0 \times 10^{-4}\left(\frac{\mathrm{GeV}}{E_{0}}\right)\left(\frac{\mathrm{erg} \mathrm{cm}^{-3}}{U_{\mathrm{ph}}+U_{\mathrm{B}}}\right) \mathrm{yr}$.

Assuming that the cosmic-ray electrons emit most of their energy in synchrotron emission at the critical frequency, the electron energy can be expressed by:

$E=\left(\frac{v B}{(16.1 \mathrm{MHz})(\mu \mathrm{G})}\right)^{1 / 2} \mathrm{GeV}$.

To calculate the electron lifetime in the filaments we use $U_{\mathrm{B}}=1.0 \times 10^{-10} \mathrm{erg} \mathrm{cm}^{-3}$ for $B=50 \mu \mathrm{G}$ and $U_{\mathrm{ph}}=4.6 \times$ $10^{-11} \mathrm{erg} \mathrm{cm}^{-3}$. We estimated the latter from the IRAS FIR flux densities of the nucleus at a height of $300 \mathrm{pc}$ using cylindrical 
Table 3. Equipartition magnetic field strengths in various regions.

\begin{tabular}{lccc}
\hline \hline Region & $\begin{array}{c}B_{\text {tot }} \\
{[\mu \mathrm{G}]}\end{array}$ & $\begin{array}{c}B_{\text {ord }} \\
{[\mu \mathrm{G}]}\end{array}$ & $\begin{array}{c}\text { Pol. degree } \\
{[\%]}\end{array}$ \\
\hline Nuclear region & $160 \pm 20$ & $<5$ & $<0.2$ \\
Filaments F1+F2 & $46 \pm 10$ & $21 \pm 5$ & $21 \pm 7$ \\
Eastern bar & $24 \pm 5$ & $11 \pm 3$ & $22 \pm 7$ \\
Western bar & $28 \pm 6$ & $5 \pm 2$ & $4 \pm 1$ \\
\hline
\end{tabular}

Notes. The polarization degree of the synchrotron emission refers to $\lambda 3 \mathrm{~cm}$, assuming $30 \%$ thermal contribution.

symmetry. The IC and synchrotron losses are therefore comparable. The electron lifetimes are $2.5(\lambda 20 \mathrm{~cm}), 1.4(\lambda 6 \mathrm{~cm})$, and $1.1 \times 10^{6} \mathrm{yr}(\lambda 3 \mathrm{~cm})$.

For dominating radiation losses, the cosmic-ray electron scaleheight is a function of the frequency

$h_{\mathrm{e}} \propto v^{-(1-a) / 4} \quad$ (diffusion)

$h_{\mathrm{e}} \propto v^{-0.5} \quad$ (convection),

depending on whether we have diffusion or convection as the transport mechanism. These scaleheights are derived as follows. We note that in the equipartition case we have $h_{\mathrm{e}}=2 h_{\text {syn }}$ (Eq. (11) in Paper I). For diffusion, $a$ is the energy dependency of the diffusion coefficient, usually assumed to be $a \approx 0.5$ for Kolgomorov type turbulence (Schlickeiser 2002). For the case of convection, the scaleheight is proportional to the electron lifetime with $t_{\mathrm{e}} \propto v^{-1 / 2}$. In the case of diffusion we have $h_{\mathrm{e}}^{2} / t_{\mathrm{e}}=\kappa$, and with $\kappa \propto E^{a}$ and $t_{\mathrm{e}} \propto 1 / E$ we get the above relation.

The observed frequency dependence of the scaleheight in the filaments is $h_{\mathrm{e}} \propto v^{0 \pm 0.16}$, which is not consistent with convection in a galactic wind. Diffusion along the filaments would require $a=1 \pm 0.64$, which can not be excluded a priori. However, diffusion should result in a Gaussian intensity profile, while the observed one is clearly exponential. Furthermore, the filament width of $\approx 300 \mathrm{pc}$ (Sect. 3.4) is larger than its scaleheight. If cosmic-ray transport is responsible for the shape of the filaments, we would expect that the filament scaleheight is greater than the filament width, because diffusion along the field lines is much faster than perpendicular to them. We conclude that the cosmicray electrons do not propagate along the filaments, if radiation losses dominate. We give an alternative explanation in Sect. 5.3.

The filaments have a Gaussian profile, as expected for diffusion, and their widths were determined in Sect. 3.4 as a function of frequency as $\propto v^{-0.13 \pm 0.18}$. This is consistent with diffusion perpendicular to the filaments with $a=0.5 \pm 0.7$, close to the expected behaviour, albeit with a large error interval. It seems therefore straightforward to assume that the cosmic-ray electrons of the surrounding medium diffuse from outside into the filaments. This explains why the filaments have a scaleheight nearly independent of the frequency, but their width decreases with increasing frequency (but see also Sect. 5.3). Summarizing, we can explain the observations by magnetic filaments with a width of about $40 \mathrm{pc}$ (or less) at the boundary of both outflow cones. The perpendicular diffusion coefficient across the filaments is $\kappa_{\perp}=1.5 \times 10^{28} \mathrm{~cm}^{2} \mathrm{~s}^{-1} \cdot E(\mathrm{GeV})^{0.5 \pm 0.7}$.

We can compare this result with theoretical expectations as the recent paper by Shalchi et al. (2010), who used numerical calculations to get an estimate for the perpendicular diffusion coefficient in a turbulent interstellar medium. Our cosmic-ray electron energy is a few $\mathrm{GeV}$, so that for protons the magnetic rigidity $R=(p \cdot c) /(Z \cdot e)$ is $R \approx 5 \times 10^{12}$ Volt. For this rigidity
Shalchi et al. (2010) give a perpendicular diffusion coefficient of $\kappa_{\perp}=3 \times 10^{27} \mathrm{~cm}^{2} \mathrm{~s}^{-1}$, a factor of 10 smaller than what we find. However, the theoretically expected parallel diffusion coefficient is a factor of 100 larger. A small amount of turbulence in the filaments is able to explain our observations.

\subsection{Energetics in the nuclear outflow}

The nuclear outflow in NGC 253 appears to be a powerful version of the outflow in our own Milky Way. The supernova rate in NGC 253 lies between 0.03 and $0.3 \mathrm{yr}^{-1}$ (Ulvestad \& Antonucci 1997; Lenc \& Tingay 2006) and is thus much larger than for the Galactic centre with $(0.02-0.08) \times 10^{-2} \mathrm{yr}^{-1}$ (Crocker et al. 2011a). Assuming that the outflow is driven by the starburst, the energy input in NGC 253 is thus a factor of 100 higher than in the Milky Way. Nevertheless, the Milky Way is able to launch a powerful galactic wind from its central region, as indicated by the strong deficiency in the radio emission compared to the FIR luminosity (Crocker et al. 2011b). Also, the gamma ray emission is deficient compared to the star formation and supernova rates, which can be explained by the advection of cosmic rays in the wind, escaping into the halo.

Dobler \& Finkbeiner (2008) have detected a microwave haze in WMAP observations towards the Galactic centre, which they interpreted as non-thermal synchrotron emission from cosmicray electrons. FERMI-LAT observations have revealed two giant gamma-ray bubbles extending to a Galactic latitude of $50^{\circ}$, and containing an energy of $10^{54}-10^{55} \mathrm{erg}$ (Dobler et al. 2010; Su et al. 2010). These discoveries were preceded by the finding of a bipolar outflow in X-ray emission by Bland-Hawthorn \& Cohen (2003), estimating a similar amount of energy released. To create these bubbles, the current release of energy by star formation is not enough. However, the bubbles have an age of $10^{7} \mathrm{yr}$, so that a starburst in the last $10 \mathrm{Myr}$ could explain them. The alternative possibilities are past accretion events of tidal debris of disrupted stars by the central black hole, which can also explain the presence of hot X-ray emitting gas (Totani 2006).

In the Galactic centre there are lobes of radio continuum emission with a diameter of $110 \mathrm{pc}$ and a height of $165 \mathrm{pc}$ (Law 2010). The Galactic centre lobes look very similar to the structure we observe in NGC 253, as can be seen by comparing Fig. 1 in Law (2010) with our Fig. 4, although the filaments in NGC 253 are a factor of 2 larger. The equipartition field strengths between 40 and $100 \mu \mathrm{G}$ are similar between the two galaxies. We note that we can trace the extension of the filaments in NGC 253 to a much larger height of 600 pc (Fig. 7), likely because our beam size of $150 \mathrm{pc}$ is much larger than that of Law (2010). Observations of the Faraday rotation favour a strong vertical magnetic field in the Galactic centre lobe region (Law et al. 2011).

We conclude that the radio continuum morphology of the Galactic centre and the nuclear starburst in NGC 253 are similar, despite their current difference in star formation rate. As the nuclear outflow in NGC 253 can be explained by the starburst model alone, the similarity may hint that the Milky Way bipolar outflow seen in X-ray and gamma-ray emission is also generated by a past starburst, rather than the past activity of the Galactic central black hole. Gamma rays are now also detected from the nucleus in NGC 253 by H.E.S.S., a Čerenkov air shower telescope (Acero et al. 2009). According to their results, the nucleus is not a good "calorimeter", indicating a loss of cosmic rays, possibly enlightening a bipolar outflow seen in gamma rays as in the Milky Way. 


\subsection{Radio-FIR correlation}

As derived from IRAS flux densities, the nucleus of NGC 253 has a FIR luminosity of FIR $/\left(3.75 \times 10^{12} \mathrm{~Hz}\right)=4.9 \times$ $10^{-8} \mathrm{erg} \mathrm{s}^{-1} \mathrm{~cm}^{-2}$. Converting this into the radio (spectral) luminosity at $1.4 \mathrm{GHz}$, using the radio-FIR correlation (e.g., Condon et al. 2002), we find $S_{1.4 \mathrm{GHz}}=6.5 \mathrm{Jy}$. Our observed radio luminosity of the nucleus of $2 \mathrm{Jy}$ (Paper I) thus falls a factor of 3 short of the expectation. This implies that the nucleus is not a "calorimeter" in the radio regime (Völk 1989), and that nonsynchrotron losses of the cosmic-ray electrons could be important.

Such a process, which we expect to be strong in a galactic wind, is adiabatic cooling in an accelerated flow. If cosmic rays are advectively transported, the adiabatic loss time is

$t_{\mathrm{ad}}=3\left(\frac{\mathrm{d} v}{\mathrm{~d} z}\right)^{-1}$,

where $\mathrm{d} v / \mathrm{d} z$ is the velocity gradient in the vertical direction. We note that the adiabatic loss time does not depend on the observing frequency, in contrast to synchrotron and IC losses. If adiabatic losses are dominating, we could naturally explain the independence of the synchrotron scaleheight from the observing frequency (Sect.3.4). As an upper limit for $t_{\mathrm{ad}}$ we take the cosmic-ray electron lifetime at $\lambda 3 \mathrm{~cm}$ of $1 \mathrm{Myr}$, as derived in Sect. 5.1. We use a cosmic-ray bulk speed of $300 \mathrm{~km} \mathrm{~s}^{-1}$ as derived from the halo scaleheights (Paper I), which was confirmed by Zirakashvili \& Völk (2006) for the nuclear outflow. Assuming linear acceleration (with respect to $z$ ), we find that the cosmic rays are accelerated between 0 and $100 \mathrm{pc}$ height.

We can also estimate the electron scaleheight as $h_{\mathrm{e}}=v t_{\mathrm{ad}}$ in the advection flow. The derived value of $h_{\mathrm{e}}=300 \mathrm{pc}$ fits to the synchrotron scaleheight of $150 \mathrm{pc}$, if the magnetic field scaleheight is much larger, such as the $600 \mathrm{pc}$ expected for energy equipartition. Since our estimated electron scaleheight fits the observations, the electron lifetime of $1 \mathrm{Myr}$ is realistic. In this case, the adiabatic losses would be comparable to the combined synchrotron and IC losses. As the last two have about the same magnitude (Sect. 5.1), we can explain a radio deficiency of a factor of 3 . We conclude that adiabatic losses in the nuclear outflow can explain the dim radio luminosity of the nucleus. But other processes may still contribute, such as ionization and bremsstrahlung losses of the cosmic rays in the nucleus (Lacki et al. 2011).

\subsection{Collimating the nuclear outflow}

The SE outflow cone has an opening angle of $26^{\circ}$ as $\mathrm{H} \alpha$ and X-ray data show (Strickland et al. 2000; Bauer et al. 2007). The X-ray data from the NW cone suggest a similarly small value although with a larger uncertainty. Interaction with the surrounding interstellar medium from the galactic disc can explain such a small cone opening angle. Figures 3 and 4 show that the filaments of the radio emission border the outflow cones visible in the hot X-ray emitting gas. The $\mathrm{H} \alpha$ emitting gas borders again the X-ray outflow. In Fig. 17 we present a multi-wavelength composite of the central region of NGC 253, which nicely shows the interplay between the different components of the interstellar medium. The radio filaments clearly surround the outflow cones at least near the base. Therefore, it is useful to ask whether the magnetic field can collimate the outflow cone?

To answer this question we make an estimate of the energy densities. Bauer et al. (2007) obtained an electron density of $n_{\mathrm{e}}=0.025 \mathrm{~cm}^{-3}$ at a temperature of $0.38 \mathrm{keV}$ in the SE cone, resulting in an energy density of $1.5 \times 10^{-11} \mathrm{erg} \mathrm{cm}^{-3}$. The NW cone is brighter with a temperature of $0.54 \mathrm{keV}$ so that here the energy density is $3.0 \times 10^{-11} \mathrm{erg} \mathrm{cm}^{-3}$ (assuming also an increased electron density). The minimum estimate of the magnetic field strength in the filaments is $50 \mu \mathrm{G}$ resulting in an energy density of $1.0 \times 10^{-10} \mathrm{erg} \mathrm{cm}^{-3}$. This is higher than the thermal energy density, so that the magnetic field can explain the collimation of both outflow cones.

\subsection{Galactic dynamo}

The parity of the magnetic fields can be used to test models of the galactic dynamo. The expectation is that a mean-field dynamo operating in a thin disc gives rise to a quadrupolar, even-parity magnetic field, where the direction of the azimuthal magnetic field is the same above and below the plane, but the direction of the vertical magnetic field component reverses with respect to the plane (e.g., Krause et al. 1989b; Haverkorn \& Heesen 2011). This also applies to a thin disc embedded in a quasi-spherical halo (Moss \& Brandenburg 1992). However, a dynamo operating in a quasi-spherical halo should give dipolar, odd-parity magnetic fields, with an azimuthal magnetic field with reversing direction across the Galactic plane, while the vertical field is directed in the same sense above and below the plane (Sokoloff \& Shukurov 1990). So far the observational evidence prefers even field parities in galaxies, based on the RM patterns in the disc (e.g., Krause et al. 1989a,b; Tabatabaei et al. 2008) or the asymmetry of polarized emission (Braun et al. 2010). These RM measurements of moderately inclined galaxies are, however, no stringent clue, because the RM structures of an even and odd parity in the disc fields are very similar in our synthetic polarization and RM maps (Figs. A.1 and A.2 in Paper II).

RM measurements in halo magnetic fields, which allow better distinction, are more difficult due to sensitivity and overlap with the disc field. Such measurements do also favour quadrupolar, even-parity fields (Soida et al. 2011). In Paper II we argued that NGC 253 is another candidate of an even disc field, albeit limited by the low resolution. This paper gives much better evidence for an even field in the disc beyond about $2 \mathrm{kpc}$ radius. High spatial resolution is crucial to distinguish between even and odd parity of the disc magnetic field. The synchrotron scaleheight of the thin disc is about $300 \mathrm{pc}$ (Paper I), so that we need a comparable resolution, like $6^{\prime \prime}(D=10 \mathrm{Mpc})$, to resolve an $\mathrm{RM}$ reversal across the midplane. Such high angular resolutions with sufficient $S / N$ in polarization now become feasible with the advent of new broadband correlators at various radio interferometers. We expect that future observations will be better able to detect odd disc fields in galaxies, some of which may have so far been classified as even.

We detected anisotropic magnetic fields as the dominant contribution to the ordered disc field observed in polarized intensity within a radius of $\approx 2 \mathrm{kpc}$. Anisotropic magnetic fields can be generated from random, isotropic fields by shear and compression. M31 is a galaxy that is dominated by a coherent axisymmetric magnetic field field from its RM structure (Berkhuijsen et al. 2003). In contrast, M 51, a grand-spiral galaxy with a prominent spiral magnetic field, shows almost no large-scale RM pattern (Fletcher et al. 2011). This galaxy is a prime example of an anisotropic disc magnetic field. The latetype spiral galaxy NGC 6946 has both regular magnetic fields between the spiral arms, in the so-called "magnetic arms", and anisotropic magnetic fields (Beck 2007). Barred galaxies, such as NGC 1097 and NGC 1365, with their strong shearing flows 


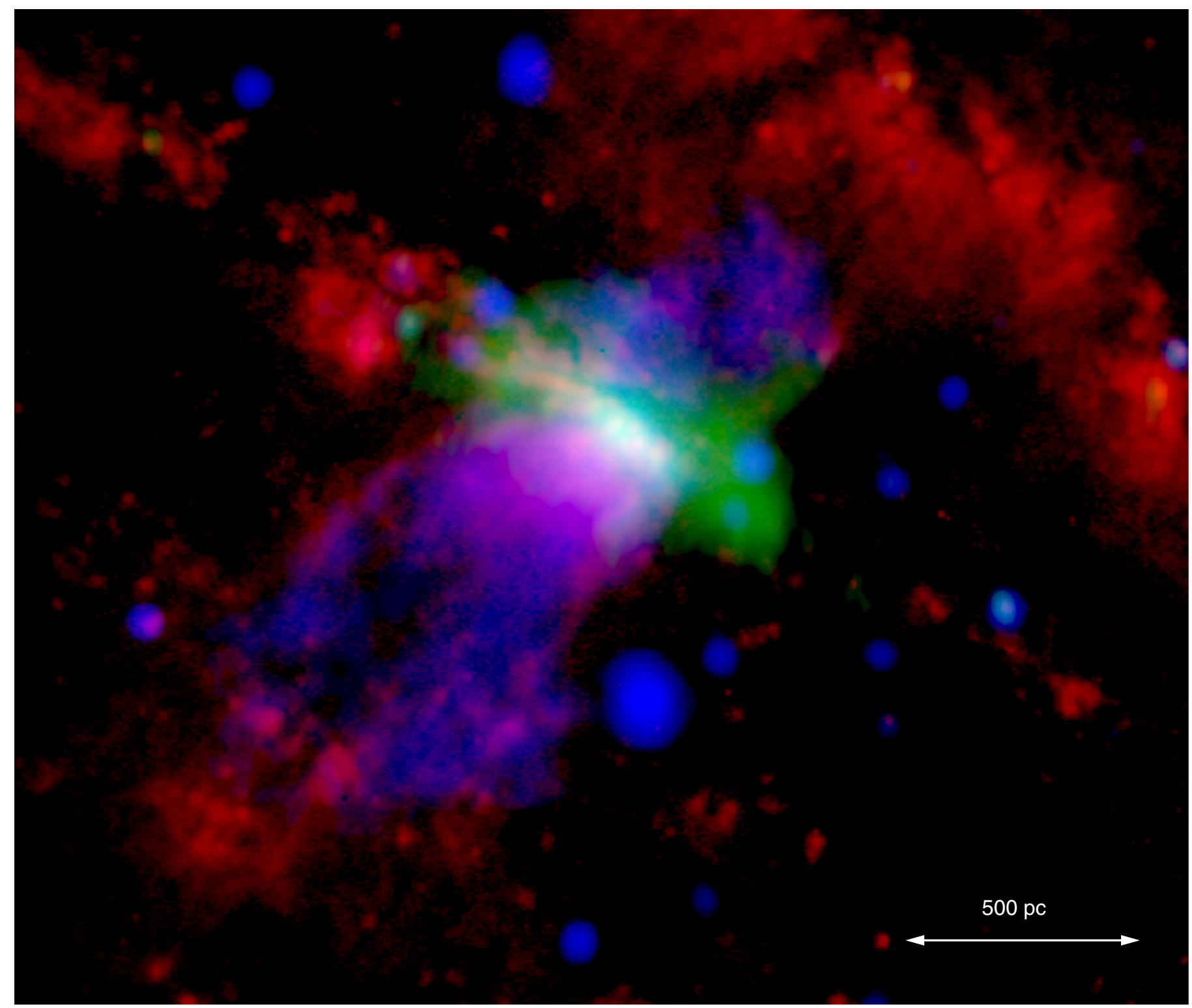

Fig. 17. Three-colour composite, multi-wavelength view of the central region in NGC 253. Red, green, and blue indicate H $\alpha$ from Westmoquette et al. (2011), $\lambda 20 \mathrm{~cm}$ radio continuum, and Chandra soft X-ray (Hardcastle, priv. com.), respectively.

host dominant anisotropic fields (Beck et al. 2005). In light of these results, it comes as no surprise that the ordered disc field in NGC 253 is anisotropic within $\approx 2 \mathrm{kpc}$ radius, where its bar resides (Sect. 3.1).

\subsection{Helical magnetic field in the outflow cone}

The helical field can be explained with help of trajectories of particles in the outflow. The conductivity of the interstellar medium is very high, so that the magnetic field lines are essentially frozen into the plasma. The field lines are taken with the gas in the outflow similar to the solar wind (Parker 1958; Weber \& Davis 1967). The magnetic field is anchored in the disc between a radius of about 130-170 pc (Fig. 15). The cone opening angle of $26^{\circ}\left(45^{\circ}\right.$ at larger heights) means that the field is transported to larger radii farther away from the disc. If we assume that no significant interaction with the surrounding medium occurs, the angular momentum of the plasma in the outflow is conserved. From the perspective of an outflowing particle, the anchor point rotates faster than the particle. The trajectories of the particles can be described by a spiral, similar to water drops in a garden sprinkler. The spiral orientation is given by the sense of rotation and is identical to that of the spiral arms, i.e. trailing arms. The vertical component of the magnetic field has to point away from the disc in order to explain the observed RM structure, which agrees with our results in Paper II for the SE halo.

We can compare the actual shape of our helix with this model. The rotation curve of the galaxy is rising linearly out to a radius of $300 \mathrm{pc}$, where it tailors off to a rotation speed of about $200 \mathrm{~km} \mathrm{~s}^{-1}$ (Sorai et al. 2000). The rotation speed at the anchor point of the filaments is thus $\approx 100 \mathrm{~km} \mathrm{~s}^{-1}$, resulting in a rotation period of $0.9 \times 10^{7} \mathrm{yr}$. We measured that the azimuthal component is about equal to the vertical component at a height of $1200 \mathrm{pc}$ (Sect. 4.5). The circumference at $150 \mathrm{pc}$ radius is $940 \mathrm{pc}$, similar to the height, so that we can expect an equal strength of the vertical and azimuthal components. During one rotation period, the particles in the outflow should thus be transported to a height of $1200 \mathrm{pc}$. The outflow speed in the nuclear outflow is 
$\approx 300 \mathrm{~km} \mathrm{~s}^{-1}$ (Westmoquette et al. 2011) at a reference height of $1 \mathrm{kpc}$, so that assuming constant acceleration the average speed is $150 \mathrm{~km} \mathrm{~s}^{-1}$. To reach a height of $1200 \mathrm{pc}$ a particle would take a time of $0.8 \times 10^{7} \mathrm{yr}$, which is in good agreement with the rotation period.

Our simple model of the magnetic field lines frozen into the outflow plasma can therefore explain the observed winding-up of the field lines into a helix. We also note that the Alfvén speed $v_{\mathrm{A}}=B / \sqrt{4 \pi \rho}$ is $30 \mathrm{~km} \mathrm{~s}^{-1}$ with $n_{\mathrm{e}}=2 \mathrm{~cm}^{-3}$ and $B=21 \mu \mathrm{G}$. This is only $10 \%$ of the outflow speed at a reference height of $1 \mathrm{kpc}$, so that the Alfvénic point would be with $100 \mathrm{pc}$ close to the disc. The stiff field lines co-rotate with the disc within the Alfvénic point. This means that the field lines start almost immediately winding up in the outflow, which accords with our observations.

Although the nuclear outflow in NGC 253 is thought to be driven entirely by a starburst and not by an active galactic nucleus (AGN, Brunthaler et al. 2009), it is worthwhile briefly comparing the outflow structure with those of AGNs. There are a number of radio polarimetric studies of AGNs, employing very long baseline interferometry and studying their magnetic fields using the synchrotron emission of highly energetic electrons in the jet (e.g., Gabuzda et al. 1992, 2000). They show that the magnetic field orientations are mainly transversal to the jet direction. This is thought to be caused by compression in shock waves, where the cosmic-ray electrons are accelerated, and thus illuminate the "knots" in the jets where the shocks occur. However, the alternative explanation is that the transverse fields are generated by the differential rotation in the accretion disc around the black hole (e.g., Contopoulos et al. 2009). This would result in a helical magnetic field farther away from the accretion disc.

One signature of a helical magnetic field in the jet of AGNs should be a reversal of the RM across the jet, as observed by us in the nuclear outflow of NGC 253. Studies of the RM in jets of AGNs are notoriously difficult, given that the jets are mostly only barely resolved and the thermal electron densities are low (Gabuzda et al. 2004; Kharb et al. 2009; O'Sullivan \& Gabuzda 2009). However, these authors find a strict dependence of the polarization angle on $\lambda^{2}$, suggesting that the jets form a Faraday screen and that the observed RM gradients are genuine and not instrumental. If the view onto the jet is nearly "side-on" $\left(90^{\circ}\right.$ to its axis) in its rest frame, the magnetic field would be transversal. If the jet is observed at a different angle to $90^{\circ}$, we should see a central region with a transversal magnetic field, whereas the field should be along the jet direction at the boundary. Such "spine + sheat" structures have been indeed observed (e.g., Attridge et al. 1999; Pushkarev et al. 2005), further supporting the existence of helical fields in AGN jets.

\section{Conclusions}

We used polarimetric radio continuum observations to study the nuclear outflow in NGC 253. Four prominent filaments were detected, which we interpreted as the walls of the two nuclear outflow cones seen in projection. The boundary of the NW outflow cone is not seen in $\mathrm{H} \alpha$ and $\mathrm{X}$-ray emission, probably due to heavy absorption. These are our main conclusions

1. The filaments have a scaleheight of $150 \pm 20 \mathrm{pc}$, independent of wavelength. The cosmic-ray electron lifetime changes by a factor of 2.3 between $\lambda \lambda 20$ and $3 \mathrm{~cm}$, so that the scaleheight should change if the electrons are subjected to dominant radiation losses. Dominating adiabatic losses could explain this behaviour, where cosmic rays and magnetic fields are advected together in the accelerating nuclear outflow.

2. The filaments have a smaller perpendicular extent for smaller electron lifetimes, so that perpendicular electron diffusion plays a role. This suggests that we are looking at filamentary magnetic fields. The perpendicular diffusion coefficient is $\kappa_{\perp}=1.5 \times 10^{28} \mathrm{~cm}^{2} \mathrm{~s}^{-1} \cdot E(\mathrm{GeV})^{0.5 \pm 0.7}$.

3. The filaments have a total magnetic field strength of $B_{\text {tot }}=$ $46 \pm 10 \mu \mathrm{G}$ and ordered field strength of $B_{\text {ord }}=21 \pm 5 \mu \mathrm{G}$. The field strengths could be higher, if the cosmic rays are not in equipartition with the magnetic field.

4. The magnetic field strength in the filaments is high enough to counteract the pressure in the nuclear outflow. The magnetic field can thus collimate the outflow and explain the small opening angle of $\approx 26^{\circ}$ by interaction with the interstellar medium. The magnetic field is aligned along the filaments, as expected for the case of compression due to a lateral pressure gradient.

5. In the SE outflow cone, the magnetic field points away from the disc in the form of a helix. The magnetic field is frozen into the plasma of the nuclear outflow, so that the angular momentum is conserved and the field wound up.

6. The ordered magnetic field in the disc observed in polarized intensity is anisotropic within a radius of about $2 \mathrm{kpc}$. At larger radii a regular field with an even axisymmetric pattern is found, as already deduced in Paper II.

Acknowledgements. V.H. is funded as a postdoctoral research assistant by the Science and Technology Facilities Council (STFC) under a rolling grant. R.J.D. is supported by the Deutsche Forschungsgemeinschaft $(D F G)$ in the framework of the research unit FOR 1048. We also acknowledge support from the DFG research unit FOR 1254. This work benefits from various ancillary data provided kindly by colleagues. We thank Kazushi Sakamoto for providing us the CO map, Charles Hoppes and Mark Westmoquette for the $\mathrm{H} \alpha$ maps, and Martin Hardcastle for the Chandra X-ray map. We thank Uli Klein for corrections to the manuscript at an early stage. We are grateful to Elias Brinks for carefully reading the manuscript. Wolfgang Reich is thanked for many useful suggestions that helped to improve the paper.

\section{Appendix A: Synthetic polarization maps}

In Heesen et al. (2009b) we used a conical halo field with a filled cone while in this paper we use a conical field that is only different from zero inside the walls of the outflow as shown in Fig. 15. The following equations are therefore identical for both cases. We stress that, even though we denote the vertical magnetic field component with the superscript "halo", this field is not a general large-scale halo field but is only concentrated in the filaments. We do not use any turbulent magnetic field, but instead restrict ourselves to ordered magnetic fields.

We integrate the source structure in a three-dimensional volume with Cartesian coordinates $(x, y, z)$, where the $x$-axis is parallel to RA, $y$ is parallel to the line-of-sight, and $z$ parallel to Dec. We also define the coordinates $\left(x^{\prime}, y^{\prime}, z^{\prime}\right)$ that are the Cartesian coordinates with respect to the galaxy. $x^{\prime}$ is parallel to the major axis, $y^{\prime}$ is parallel to the minor axis, and $z^{\prime}$ parallel to the rotation axis. We can relate both coordinate systems by linear transformations first rotating around the inclination angle $i$ and then rotating it around the position angle. For convenience we use $\iota=90^{\circ}-i$, which is the angle between $y$ and $y^{\prime}$, and $\zeta=90^{\circ}-$ PA which is the angle between $z$ and $z^{\prime}$. The reference coordinates $\left(x_{\text {ref }}, y_{\text {ref }}, z_{\text {ref }}\right)$ are the central coordinates of the galaxy. Moreover, we use cylindrical coordinates for the observer $(\rho, \phi, z)$ and in the galaxy $\left(\rho^{\prime}, \phi^{\prime}, z^{\prime}\right)$. 


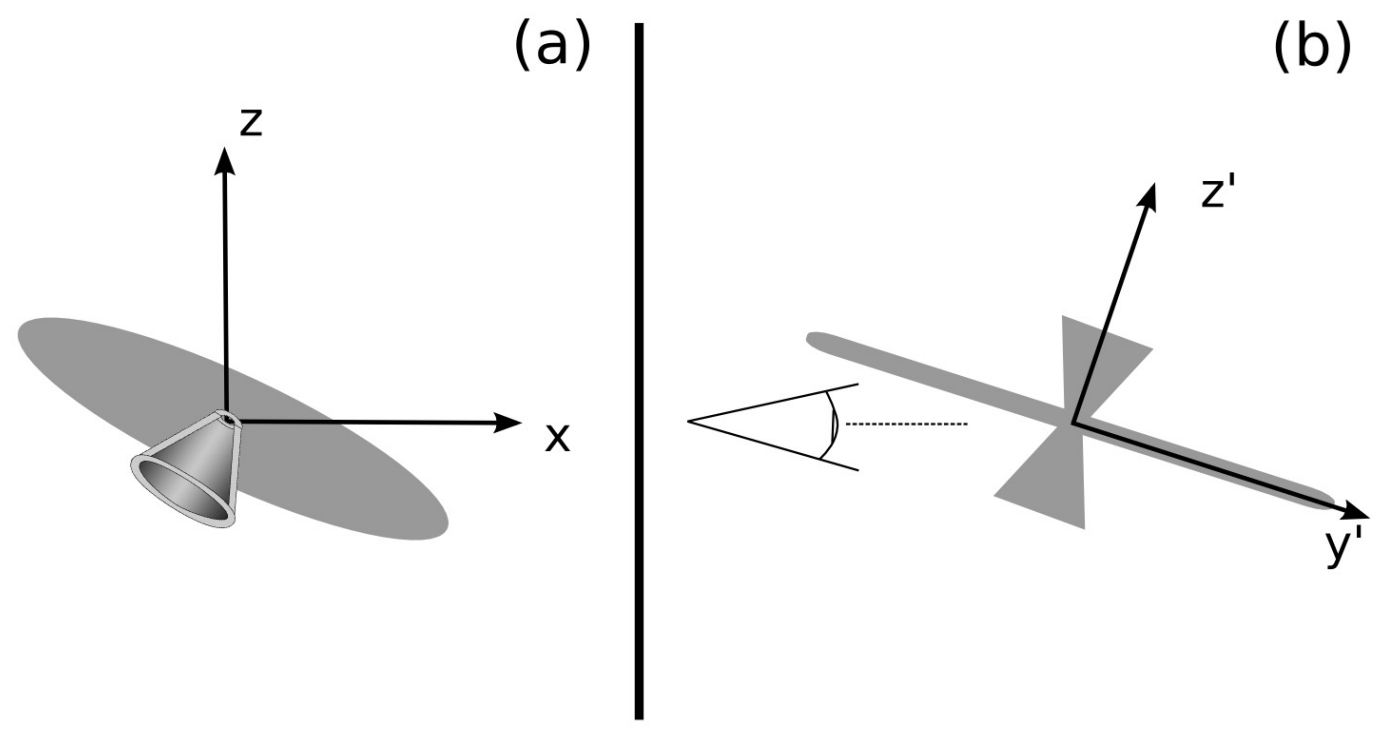

Fig. A.1. Coordinates used for the synthetic polarization maps. a) View along the line-of-sight in the direction of the $y$-axis. b) View from the side in the opposite direction to the $x^{\prime}$-axis, where the observer is located at the left.

The frame of reference Cartesian coordinates of the galaxy can be expressed in the observer's as

$$
\begin{aligned}
x^{\prime}= & \cos (\zeta)\left(x-x_{\mathrm{ref}}\right)-\sin (\zeta)\left(z-z_{\mathrm{ref}}\right) \\
y^{\prime}= & -\sin (\zeta) \sin (\iota)\left(x-x_{\mathrm{ref}}\right)+\cos (\iota)\left(y-y_{\mathrm{ref}}\right) \\
& -\sin (\iota) \cos (\zeta)\left(z-z_{\mathrm{ref}}\right) \\
z^{\prime}= & \sin (\zeta) \cos (\iota)\left(x-x_{\mathrm{ref}}\right)+\sin (\iota)\left(y-y_{\mathrm{ref}}\right) \\
& +\cos (\iota) \cos (\zeta)\left(z-z_{\mathrm{ref}}\right)
\end{aligned}
$$

For the strength of the magnetic field in the disc we use a Gaussian profile with respect to the galactocentric radius and an exponential vertical decrease with a scaleheight $h_{\mathrm{B} \text {,disc }}$. For NGC 253 we used a two-component Gaussian distribution as a function of $\rho^{\prime}$, which is not shown here for simplicity. The parameter $\sigma$ can be expressed by the full width half mean (FWHM) as $\sigma=\mathrm{FWHM} /(2 \sqrt{2 \log (2)})$ in this example and all following cases where we use a Gaussian distribution:

$B^{\text {disc }}=B_{0}^{\text {disc }} \exp \left(\frac{-\left|z^{\prime}\right|}{h_{\mathrm{B}}^{\text {disc }}}\right) \exp \left(\frac{-\rho^{\prime 2}}{2 \sigma_{\text {disc }}^{2}}\right)$,

where we take the absolute value $\left|z^{\prime}\right|$ of the vertical distance to the galactic midplane. The magnetic field strength in the halo (filament) can be expressed as the disc field by

$B^{\text {halo }}=B_{0}^{\text {halo }} \exp \left(\frac{-\left|z^{\prime}\right|}{h_{\mathrm{B}}^{\text {halo }}}\right) \exp \left(\frac{-\rho^{\prime 2}}{2 \sigma_{\text {halo }}^{2}}\right)$.

Here, $B_{0}^{\text {disc }}$ and $B_{0}^{\text {halo }}$ are the strengths of the ordered field in the disc and halo. The $z^{\prime}$-component of the halo (filament) field is

$B_{z \text {,halo }}^{\prime}= \pm \frac{B_{\text {halo }}^{\prime}}{\sqrt{1.0+\tan ^{2}(\alpha)+\tan ^{2}(\beta)}}$,

where for even parity " + " is used if $z^{\prime} \leq 0$ and for odd parity "+" is used for all $z^{\prime}$. We can now derive the $\phi^{\prime}$-component as

$B_{\phi^{\prime}, \text { halo }}^{\prime}= \pm B_{z \text {,halo }}^{\prime} \tan (\alpha)$, where "+" is used if $z^{\prime} \leq 0$. The angle $\alpha$ gives the azimuthal component of the halo (filament) field and $\beta$ is the angle with respect to the rotation axis, i.e. half of the cone opening angle. Now

$B_{\rho^{\prime}, \text { halo }}^{\prime}= \pm B_{z \text {,halo }}^{\prime} \tan (\beta)$,

where "+" is used if $z^{\prime} \leq 0$. Now

$B_{\phi^{\prime}, \mathrm{disc}}^{\prime}= \pm \frac{B_{\mathrm{disc}}^{\prime}}{1.0+\tan ^{2}(\chi)}$,

where for even parity "-" is used for all $z$ ' and for odd parity "+" is used if $z^{\prime} \leq 0$. The azimuthal disc component is

$B_{\rho^{\prime}, \mathrm{disc}}= \pm \tan (\chi) B_{\phi^{\prime}, \mathrm{disc}}$

where - is used for an inwards pointing field. For the following we define $\varphi$ as

$\varphi=\operatorname{atan} 2\left(y^{\prime}, x^{\prime}\right)$

where the numerical function "atan2" is equivalent to atan, but returns the angle in all four quadrants $\left(360^{\circ}\right)$ rather than only between $-90^{\circ}$ and $90^{\circ}$. Transforming back from the cylindrical coordinate system of the galaxy into the Cartesian coordinate system of the observer we first need to transform to the Cartesian system in the galaxy:

$\left(B_{x}^{\mathrm{disc}}\right)^{\prime}=\left(B_{\rho}^{\mathrm{disc}}\right)^{\prime} \cos (\varphi)-\left(B_{\phi}^{\mathrm{disc}}\right)^{\prime} \sin (\varphi)$

$\left(B_{y}^{\mathrm{disc}}\right)^{\prime}=\left(B_{\rho}^{\mathrm{disc}}\right)^{\prime} \sin (\varphi)+\left(B_{\phi}^{\mathrm{disc}}\right)^{\prime} \cos (\varphi)$.

Transforming above equations into the Cartesian system of the observer,

$$
\begin{aligned}
B_{x}^{\mathrm{disc}}= & \cos (\zeta)\left(B_{x}^{\mathrm{disc}}\right)^{\prime}-\sin (\zeta) \sin (\iota)\left(B_{y}^{\mathrm{disc}}\right)^{\prime} \\
& +\sin (\zeta) \cos (\iota)\left(B_{z}^{\mathrm{disc}}\right)^{\prime} \\
B_{y}^{\mathrm{disc}}= & \cos (\iota)\left(B_{y}^{\mathrm{disc}}\right)^{\prime}+\sin (\iota)\left(B_{z}^{\mathrm{disc}}\right)^{\prime}
\end{aligned}
$$


V. Heesen et al.: Helical magnetic fields in the nuclear outflow of NGC 253. III.

$$
\begin{aligned}
B_{z}^{\mathrm{disc}}= & -\sin (\zeta)\left(B_{x}^{\mathrm{disc}}\right)^{\prime}-\sin (\iota) \cos (\zeta)\left(B_{y}^{\mathrm{disc}}\right)^{\prime} \\
& +\cos (\iota) \cos (\zeta)\left(B_{z}^{\mathrm{disc}}\right)^{\prime} .
\end{aligned}
$$

The same equation applies to the halo (filament) magnetic field that is not shown here. Now we have the magnetic field components in the Cartesian system of the observer. We define the local polarization angles for the disc and halo field as

$$
\begin{aligned}
& \psi_{\text {disc }}=\operatorname{atan} 2\left\{-\left(B_{x}^{\text {disc }}\right)^{\prime},\left(B_{z}^{\text {disc }}\right)^{\prime}\right\}-90^{\circ} \\
& \psi_{\text {halo }}=\operatorname{atan} 2\left\{-\left(B_{x}^{\prime \text { halo }}\right)^{\prime},\left(B_{z}^{\prime \text { halo }}\right)^{\prime}\right\}-90^{\circ} .
\end{aligned}
$$

The $90^{\circ}$ correction is to transform the polarization angle into the magnetic field orientation. The Faraday rotation shifts the polarization angle if we go a step $\Delta y$ along the line-of-sight:

$$
\begin{aligned}
\Delta \psi(y+\Delta y)= & \Delta \psi(y)-0.81 \lambda^{2} n_{\mathrm{e}} \exp \left(\frac{-\left|z^{\prime}\right|}{h_{\mathrm{e}}}\right) \exp \left(\frac{\rho^{\prime 2}}{2 \sigma_{\mathrm{e}}^{2}}\right) \\
& \times\left\{\left(\frac{B_{\text {reg }}}{B_{\text {ord }}}\right)^{\text {disc }}\left(B_{y}^{\text {disc }}\right)^{\prime}\right. \\
& \left.+\left(\frac{B_{\text {reg }}}{B_{\text {ord }}}\right)^{\text {halo }}\left(B_{y}^{\text {halo }}\right)^{\prime}\right\} \Delta y .
\end{aligned}
$$

The "-" is because we are going in positive $y$-direction, whereas the polarized wave is going from the source to the observer. The ratio of the regular to the ordered field describes the anisotropy of the magnetic field, with $B_{\text {reg }} \leq B_{\text {ord }}$.

We can now also calculate the Faraday screen RM if the galaxy would be a screen:

$$
\begin{aligned}
\operatorname{RM}_{\text {screen }}(y+\Delta y)= & \mathrm{RM}_{\text {screen }}(y)-0.81 n_{\mathrm{e}} \exp \left(\frac{-\left|z^{\prime}\right|}{h_{\mathrm{e}}}\right) \\
& \times \exp \left(\frac{\rho^{\prime 2}}{2 \sigma_{\mathrm{e}}^{2}}\right)\left\{\left(\frac{B_{\text {reg }}}{B_{\text {ord }}}\right)^{\text {disc }}\left(B_{y}^{\text {disc }}\right)^{\prime}\right. \\
& \left.+\left(\frac{B_{\text {reg }}}{B_{\text {ord }}}\right)^{\text {halo }}\left(B_{y}^{\text {halo }}\right)^{\prime}\right\} \Delta y .
\end{aligned}
$$

For the observed polarization angle we obtain with Faraday rotation included:

$\psi_{\text {disc }}^{\text {Faraday }}=\psi_{\text {disc }}+\Delta \psi$.

The cosmic-ray energy density $n_{\mathrm{CR}}$ is scaled according to the equipartition condition as

$$
\begin{aligned}
n_{\mathrm{CR}}= & n_{\mathrm{CR}, 0}\left(\frac{\left(B_{x}^{\text {disc }}\right)^{2}+\left(B_{y}^{\text {disc }}\right)^{2}+\left(B_{z}^{\text {disc }}\right)^{2}}{\left(B_{0}^{\text {disc }}\right)^{2}+\left(B_{0}^{\text {halo }}\right)^{2}}\right. \\
& \left.+\frac{\left(B_{x}^{\text {halo }}\right)^{2}+\left(B_{y}^{\text {halo }}\right)^{2}+\left(B_{z}^{\text {halo }}\right)^{2}}{\left(B_{0}^{\text {disc }}\right)^{2}+\left(B_{0}^{\text {halo }}\right)^{2}}\right) .
\end{aligned}
$$

The Stokes $Q$ integration along the line-of-sight is

$$
\begin{aligned}
Q, U(y+\Delta y)= & Q, U(y)+n_{\mathrm{CR}} \\
& \times\left\{\left(\left(B_{x}^{\text {disc }}\right)^{2}+\left(B_{z}^{\text {disc }}\right)^{2}\right) \cos \left(2 \psi_{\text {disc }}^{\text {Faraday }}\right)\right. \\
& \left.+\left(\left(B_{x}^{\text {halo }}\right)^{2}+\left(B_{z}^{\text {halo }}\right)^{2}\right) \cos \left(2 \psi_{\text {halo }}^{\text {Faraday }}\right)\right\},
\end{aligned}
$$

where for Stokes $U$, "sin" is used instead of "cos". Stokes $Q$ and $U$ have to be calculated at at least two frequencies in order to calculate the rotation measure. The two-dimensional arrays of $Q(x, z)$ and $U(x, z)$ are written out as a fits file for which we used cfitsio ${ }^{6}$.

${ }^{6}$ CFITSIO is a library of $\mathrm{C}$ and Fortran sub-routines for reading
and writing data files in FITS (Flexible Image Transport System) data

\section{References}

Acero, F., Aharonian, F., Akhperjanian, A. G., et al. 2009, Science, 326, 1080 Adriani, O., Barbarino, G. C., Bazilevskaya, G. A., et al. 2011, Phys. Rev. Lett., 106, 201101

Athanassoula, E. 1992, MNRAS, 259, 345

Attridge, J. M., Roberts, D. H., \& Wardle, J. F. C. 1999, ApJ, 518, L87

Baars, J. W. M., Genzel, R., Pauliny-Toth, I. I. K., \& Witzel, A. 1977, A\&A, 61, 99

Bauer, M., Pietsch, W., Trinchieri, G., et al. 2007, A\&A, 467, 979

Beck, R. 2007, A\&A, 470, 539

Beck, R. 2009, Ap\&SS, 320, 77

Beck, R., \& Krause, M. 2005, AN, 326, 414

Beck, R., Brandenburg, A., Moss, D., Shukurov, A., \& Sokoloff, D. 1996, ARA\&A, 34, 155

Beck, R., Fletcher, A., Shukurov, A., et al. 2005, A\&A, 444, 739

Berkhuijsen, E. M., Beck, R., \& Hoernes, P. 2003, A\&A, 398, 937

Bland-Hawthorn, J., \& Cohen, M. 2003, ApJ, 582, 246

Braun, R. 1988, Millimeter Array Memo, 46

Braun, R., Heald, G., \& Beck, R. 2010, A\&A, 514, A42

Breitschwerdt, D. 2008, Nature, 452, 826

Breitschwerdt, D., McKenzie, J. F., \& Völk, H. J. 1991, A\&A, 245, 79

Briggs, D. S. 1995, in American Astronomical Society Meeting Abstracts, BAAS, 27, 1444

Brunthaler, A., Castangia, P., Tarchi, A., et al. 2009, A\&A, 497, 103

Burn, B. J. 1966, MNRAS, 133, 67

Carilli, C. L., Holdaway, M. A., Ho, P. T. P., \& de Pree, C. G. 1992, ApJ, 399, L59

Chyży, K. T., \& Beck, R. 2004, A\&A, 417, 541

Condon, J. J. 1992, ARA\&A, 30, 575

Condon, J. J., Cotton, W. D., \& Broderick, J. J. 2002, AJ, 124, 675

Contopoulos, I., Christodoulou, D. M., Kazanas, D., \& Gabuzda, D. C. 2009, ApJ, 702, L148

Crocker, R. M., Jones, D. I., Aharonian, F., et al. 2011a, MNRAS, 413, 763

Crocker, R. M., Jones, D. I., Aharonian, F., et al. 2011b, MNRAS, 411, L11

Dalcanton, J., Williams, B., Gogarten, S., et al. 2007, BAAS, 38, 870

Dalla Vecchia, C., \& Schaye, J. 2008, MNRAS, 387, 1431

de Avillez, M. A. 2000, MNRAS, 315, 479

Dobler, G., \& Finkbeiner, D. P. 2008, ApJ, 680, 1222

Dobler, G., Finkbeiner, D. P., Cholis, I., Slatyer, T., \& Weiner, N. 2010, ApJ, 717,825

Downes, D., \& Solomon, P. M. 1998, ApJ, 507, 615

Dumas, G., Schinnerer, E., Tabatabaei, F. S., et al. 2011, AJ, 141, 41

Dumke, M., \& Krause, M. 1998, in Lecture Notes in Physics, 506, The Local Bubble and Beyond, ed. D. Breitschwerdt, M. J. Freyberg, \& J. Trümper (Berlin: Springer Verlag), IAU Colloq., 166, 555

Duric, N., Irwin, J., \& Bloemen, H. 1998, A\&A, 331, 428

Fletcher, A., Beck, R., Shukurov, A., Berkhuijsen, E. M., \& Horellou, C. 2011, MNRAS, 412, 2396

Gabuzda, D. C., Cawthorne, T. V., Roberts, D. H., \& Wardle, J. F. C. 1992, ApJ, 388,40

Gabuzda, D. C., Kochenov, P. Y., \& Cawthorne, T. V. 2000, MNRAS, 319, 1125

Gabuzda, D. C., Murray, É., \& Cronin, P. 2004, MNRAS, 351, L89

Gissinger, C., Fromang, S., \& Dormy, E. 2009, MNRAS, 394, L84

Hanasz, M., Otmianowska-Mazur, K., Kowal, G., \& Lesch, H. 2009, A\&A, 498, 335

Haverkorn, M., \& Heesen, V. 2011, Space Sci. Rev., 29

Heckman, T. M., Lehnert, M. D., Strickland, D. K., \& Armus, L. 2000, ApJS, 129,493

Heesen, V., Beck, R., Krause, M., \& Dettmar, R.-J. 2009a, A\&A, 494, 563

Heesen, V., Krause, M., Beck, R., \& Dettmar, R.-J. 2009b, A\&A, 506, 1123

Hoopes, C. G., Walterbos, R. A. M., \& Greenwalt, B. E. 1996, AJ, 112, 1429

Ipavich, F. M. 1975, ApJ, 196, 107

Jarrett, T. H., Chester, T., Cutri, R., Schneider, S. E., \& Huchra, J. P. 2003, AJ, 125,525

Karachentsev, I. D., Grebel, E. K., Sharina, M. E., et al. 2003, A\&A, 404, 93

Kennicutt, Jr., R. C. 1998, ApJ, 498, 541

Kepley, A. A., Mühle, S., Everett, J., et al. 2010, ApJ, 712, 536

Kharb, P., Gabuzda, D. C., O'Dea, C. P., Shastri, P., \& Baum, S. A. 2009, ApJ, 694,1485

Krause, M. 2008, in Magnetic Fields in the Universe II, ed. A. Esquivel, RevMexAA, 36, 25

Krause, M., Beck, R., \& Hummel, E. 1989a, A\&A, 217, 17

format. CFITSIO is free software and can be obtained at http:// heasarc.gsfc.nasa.gov/fitsio 
Krause, M., Hummel, E., \& Beck, R. 1989b, A\&A, 217, 4

Lacki, B. C., Thompson, T. A., Quataert, E., Loeb, A., \& Waxman, E. 2011, ApJ, 734, 107

Law, C. J. 2010, ApJ, 708, 474

Law, C. J., Brentjens, M. A., \& Novak, G. 2011, ApJ, 731, 36

Lenc, E., \& Tingay, S. J. 2006, AJ, 132, 1333

Mac Low, M.-M., \& Ferrara, A. 1999, ApJ, 513, 142

Moss, D., \& Brandenburg, A. 1992, A\&A, 256, 371

Norman, C. A., \& Ikeuchi, S. 1989, ApJ, 345, 372

Noutsos, A., Johnston, S., Kramer, M., \& Karastergiou, A. 2008, MNRAS, 386, 1881

Ondrechen, M. P., \& van der Hulst, J. M. 1989, ApJ, 342, 29

Ondrechen, M. P., van der Hulst, J. M., \& Hummel, E. 1989, ApJ, 342, 39

O'Sullivan, S. P., \& Gabuzda, D. C. 2009, MNRAS, 400, 26

Ott, J., Weiss, A., Henkel, C., \& Walter, F. 2005, ApJ, 629, 767

Parker, E. N. 1958, ApJ, 128, 664

Patrikeev, I., Fletcher, A., Stepanov, R., et al. 2006, A\&A, 458, 441

Pence, W. D. 1981, ApJ, 247, 473

Pietsch, W., Vogler, A., Klein, U., \& Zinnecker, H. 2000, A\&A, 360, 24

Pushkarev, A. B., Gabuzda, D. C., Vetukhnovskaya, Y. N., \& Yakimov, V. E. 2005, MNRAS, 356, 859

Reuter, H., Klein, U., Lesch, H., Wielebinski, R., \& Kronberg, P. P. 1992, A\&A, 256,10

Reuter, H., Klein, U., Lesch, H., Wielebinski, R., \& Kronberg, P. P. 1994, A\&A, 282,724

Reynolds, S. P., Gaensler, B. M., \& Bocchino, F. 2011, Space Sci. Rev., 61

Sakamoto, K., Ho, P. T. P., Iono, D., et al. 2006, ApJ, 636, 685

Schlickeiser, R. 2002, Cosmic Ray Astrophysics (Germany, Berlin: Springer)

Schulz, H., \& Wegner, G. 1992, A\&A, 266, 167

Shalchi, A., Büsching, I., Lazarian, A., \& Schlickeiser, R. 2010, ApJ, 725, 2117

Shikaze, Y., Haino, S., Abe, K., et al. 2007, Astropart. Phys., 28, 154
Soida, M., Krause, M., Dettmar, R.-J., \& Urbanik, M. 2011, A\&A, 531, A127

Sokoloff, D., \& Shukurov, A. 1990, Nature, 347, 51

Sokoloff, D. D., Bykov, A. A., Shukurov, A., et al. 1998, MNRAS, 299, 189

Sorai, K., Nakai, N., Kuno, N., Nishiyama, K., \& Hasegawa, T. 2000, PASJ, 52, 785

Strickland, D. K., Heckman, T. M., Weaver, K. A., \& Dahlem, M. 2000, AJ, 120, 2965

Strickland, D. K., Heckman, T. M., Weaver, K. A., Hoopes, C. G., \& Dahlem, M. 2002, ApJ, 568, 689

Su, M., Slatyer, T. R., \& Finkbeiner, D. P. 2010, ApJ, 724, 1044

Suchkov, A. A., Balsara, D. S., Heckman, T. M., \& Leitherner, C. 1994, ApJ, 430, 511

Tabatabaei, F. S., Krause, M., Fletcher, A., \& Beck, R. 2008, A\&A, 490, 1005

Taylor, A. R., Stil, J. M., \& Sunstrum, C. 2009, ApJ, 702, 1230

Thompson, T. A., Quataert, E., Waxman, E., Murray, N., \& Martin, C. L. 2006, ApJ, 645, 186

Totani, T. 2006, PASJ, 58, 965

Tüllmann, R., Dettmar, R.-J., Soida, M., Urbanik, M., \& Rossa, J. 2000, A\&A, 364, L36

Ulvestad, J. S., \& Antonucci, R. R. J. 1997, ApJ, 488, 621

Völk, H. J. 1989, A\&A, 218, 67

Vollmer, B., Soida, M., Beck, R., et al. 2007, A\&A, 464, L37

Vollmer, B., Soida, M., Chung, A., et al. 2010, A\&A, 512, A36

Weber, E. J., \& Davis, L. J. 1967, ApJ, 148, 217

Weiß, A., Walter, F., \& Scoville, N. Z. 2005, A\&A, 438, 533

Westmoquette, M. S., Smith, L. J., \& Gallagher, III, J. S. 2011, MNRAS, 414 3719

Weżgowiec, M., Urbanik, M., Vollmer, B., et al. 2007, A\&A, 471, 93

Wills, K. A., Redman, M. P., Muxlow, T. W. B., \& Pedlar, A. 1999, MNRAS, 309,395

Zirakashvili, V. N., \& Völk, H. J. 2006, ApJ, 636, 140 